\title{
Landau-Heisenberg Hamiltonian model for FeRh
}

\author{
P. M. Derlet* \\ Condensed Matter Theory Group, Paul Scherrer Institute, CH-5232 Villigen PSI, Switzerland
}

(Received 11 March 2012; published 25 May 2012)

\begin{abstract}
An empirical model is developed for the FeRh system with the view of gaining further insight into the first-order antiferromagnetic-ferromagnetic (AFM-FM) and volume phase transition known to occur at $370 \mathrm{~K}$. A volume-per-atom dependent minimal nearest neighbor Landau-Heisenberg Hamiltonian is employed in which longitudinal and transverse moment fluctuations are considered for both the $\mathrm{Fe}$ and $\mathrm{Rh}$ atoms. As a function of volume-per-atom, the corresponding onsite Landau function coefficients and the nearest-neighbor exchange parameters are fitted directly to a wide range of existing colinear and noncolinear density functional theory calculations. Using a developed Monte Carlo strategy the thermal properties of the AFM and FM phases are investigated, as well as the phase transition. It is found that the model is able to describe well the thermal expansion, heat capacities and the associated entropy increase that accompanies the magnetic/volume phase transition. The model suggests an equally important role for the magnetic and volume fluctuations in driving the phase transition.
\end{abstract}

DOI: 10.1103/PhysRevB.85.174431

PACS number(s): $75.10 . \mathrm{Hk}$

\section{INTRODUCTION}

$\mathrm{Fe}_{50} \mathrm{Rh}_{50}$, in the ordered cesium-chloride structure, undergoes a first-order phase transition at $370 \mathrm{~K}$ from an antiferromagnetic (AFM) to a ferromagnetic (FM) structure, ${ }^{1-3}$ and is accompanied by a volume increase of approximately $0.5 \%-1 \% .^{4}$ More recent neutron measurements demonstrate that, within experimental error, the magnetic and volume transitions occur at the same critical temperature. ${ }^{5}$ From neutron experiments, ${ }^{6}$ the high-temperature FM state is characterized by a magnetic structure in which the $\mathrm{Fe}$ moments have a magnitude of $3 \mu_{\mathrm{B}}$ and the $\mathrm{Rh}$ moments, a magnitude of $1 \mu_{\mathrm{B}}$. For the low-temperature AFM phase, the sublattice $\mathrm{Fe}$ moment magnitudes are somewhat smaller and the $\mathrm{Rh}$ has a considerably reduced moment. With the help of early electronic structure calculations, ${ }^{7-9}$ the generally agreed upon, $0 \mathrm{~K}$, magnetic configurations of both magnetic phases are shown in Fig. 1. Experimentally, the AFM to FM transition is also accompanied by a large increase in entropy, ${ }^{10-14}$ that has been associated with the different heat capacity properties of the two phases. ${ }^{15,16}$ How the lattice and magnetic (electronic/spin) degrees of freedom give rise to these quantities, and what critical role they play in the phase transition remains an contemporary question that has gained recent impetus in light of a new class of experiments able to directly probe these degrees of freedom with picosecond temporal resolution. ${ }^{17-22}$

Early local (local density approximation) density functional theory (DFT) work by Moruzzi and Marcus ${ }^{9}$ demonstrated a number of AF structures, the ground state being referred to as the AFMII phase [see Fig. 1(a)]. In this work, the magnetic energy landscape was explored with respect to colinear variations of the total magnetic moment. It was found that there also exists a metastable FM configuration and, upon a significant increase of volume-per-atom, this becomes the lowest-energy magnetic structure. Calculated equation-of-state curves revealed that a transition between these magnetic structures, at their associated equilibrium volumes, would be accompanied by a change in the volumeper-atom of approximately $1.7 \%$. In the AFMII phase, the magnetic moment magnitudes of the Fe atoms are somewhat higher than that of bulk BCC Fe at approximately $3 \mu_{\mathrm{B}}$, with the $\mathrm{Rh}$ moment being identically zero. For the FM phase, the work found that with the associated volume increase the Fe moment increases its moment magnitude to approximately $3.1 \mu_{\mathrm{B}}$ and the parallel (now nonzero) $\mathrm{Rh}$ moment magnitude is approximately $1 \mu_{\mathrm{B}}$. The energy difference between the AFMII and FM states at their corresponding equilibrium volumes was found to be approximately $2 \mathrm{mRy}$ per atom.

More recent nonlocal DFT work, by Gu and Antropov, ${ }^{23}$ using a gradient-corrected exchange correlation function found this energy difference to be much smaller at approximately $0.2 \mathrm{mRy}$ per atom, which is closer to that seen in experiment. ${ }^{24}$ The relative volume differences using nonlocal DFT did not change greatly when compared to their local DFT values, however in general the absolute equilibrium volumes increased by up to $1 \%$. In this work noncolinear magnetic configurations were also considered. Indeed a simple magnetic structural transformation was detailed linking the two phases. Here the oppositely aligned Fe moments of the AFMII phase are canted towards an orthogonal [100] direction with the Rh moment constrained to be parallel to the sum of the Fe moments. With a canting of $90^{\circ}$, the FM structure is obtained with the total magnetic moment direction being orthogonal to the sublattice moment direction of the starting AFMII magnetic structure. For volumes at, and in the vicinity of, the equilibrium volumes of the two stable phases, the FM phase is unstable with respect to this magnetic transformation when using local DFT, but is found to be stabilized when nonlocal DFT is used.

In the DFT work of Sandratskii and Mavropoulos, ${ }^{25}$ who used a local density approximation for the exchange correlation, an energy barrier was also found at a much higher volume suggesting nonlocal corrections, whilst necessary for increased accuracy, are not inherently necessary for the stabilization of the FM phase. This quite recent and pedagogical work systematically investigated a range of noncolinear variations, shedding important light on the underlying exchange mechanisms of the system, providing important data for the development of empirical models such as that reported here.

Parallel and in conjunction with the DFT work, a number of empirical/semi-empirical models have been developed based 

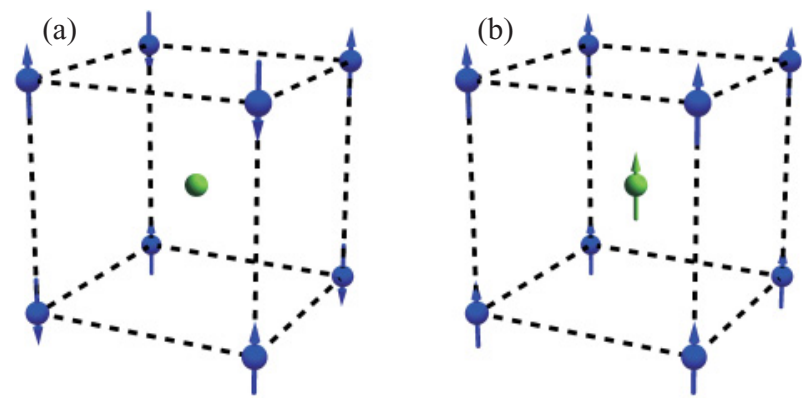

FIG. 1. (Color online) The relevant zero Kelvin colinear equilibrium magnetic structures of the FeRh system: (a) AFMII and (b) FM. In both figures, the blue (green) balls/arrows represent the $\mathrm{Fe}(\mathrm{Rh})$ atoms/moments.

on some form of exchange. An early and well-known model by Kittel, ${ }^{26}$ exploits a simple Heisenberg model, in which the exchange interaction between the $\mathrm{Fe}$ moments undergoes a sign change at the appropriate volume-per-atom, thus giving a simple explanation of the AFM to FM phase transformation. This exchange inversion model is however not considered to be appropriate for the FeRh system, since it is unable to explain the experimentally observed strong entropy change measured during the phase transition. ${ }^{13}$

The fact that at a finite temperature the FM magnetic structure becomes the stable state, and all $0 \mathrm{~K}$ DFT results observe that the AFMII phase has the lowest energy for the experimental volume-per-atom regime, points towards the FM phase being stabilized by entropy associated with either the spin-degrees of freedom and/or the lattice degrees of freedom. From the local DFT work of Ref. 9, the approximately 2 mRy energy difference between the two phases suggests a considerable entropy effect would have to be at play. However, with the nonlocal DFT work reducing this energy difference by almost an order of magnitude, the required entropy now falls into the regime that is achievable through transverse spin fluctuations. Indeed, using classical magnon theory, $\mathrm{Gu}$ and Antropov $^{23}$ have demonstrated that such spin-wave excitations can stabilize the FM phase. Their calculations were performed within the frame work of a classical Heisenberg model whose exchange constants were derived from their noncolinear DFT calculations. In their work two quite separate Heisenberg models were assumed for each phase, where in the AFM phase the Rh was not magnetic and therefore excluded, and in the FM phase, it had a fixed (volume independent) moment magnitude of $1 \mu_{\mathrm{B}}$. In this model, the Fe moments also had fixed moment magnitudes of $3 \mu_{\mathrm{B}}$. The entropy change at the transition temperature due to such spin-wave excitations was found to be comparable to experiment.

$\mathrm{Ju}$ et al. ${ }^{17}$ advanced the important step that the $\mathrm{Rh}$ atom was able to have a finite moment magnitude in the AFMII phase, due to an exchange field arising from the neighboring $\mathrm{Fe}$ atoms via a temperature-dependent susceptibility. From their DFT calculations, it was argued that the energy for a finite Rh moment depended quadratically on it's moment magnitude and, with the prefactor being negative, was interpreted within the framework of Stoner magnetism. Such a temperaturedependent term introduces an effective FM exchange interaction between the eight $\mathrm{Fe}$ atoms neighboring each $\mathrm{Rh}$ atom and thus a first, second, and third neighbor effective Fe-Fe FM exchange interaction that is linearly dependent on the proposed susceptibility. As the temperature approaches the transition temperature and the susceptibility rises, eventually this effective FM interaction competes with the explicit AFM exchange between the nearest neighbor $\mathrm{Fe}$ atoms causing the AFMII structure to be unstable and thus the AFM-FM phase transition.

On the other hand, the work of Sandratskii and Mavropoulos ${ }^{25}$ found a spatially varying spin-density at the $\mathrm{Rh}$ atom for the AFM state, which integrates to zero giving the expected zero net magnetic moment for the $\mathrm{Rh}$ atom. Such a result is at variance with a simple Stoner picture of the nonmagnetic state where the zero moment corresponds to a uniform, approximately zero, spin density. Their work also found that the $\mathrm{Rh}$ atom is able to have a finite moment magnitude, but at the expense of an energy that varied quadratically with moment magnitude. Similar to Ju et al. ${ }^{17}$ this allows for a single Heisenberg-type Hamiltonian to be applicable to both phases and therefore a study of the volume dependence of the exchange parameters. In Ref. 25, the main interactions are an Fe-Fe AFM exchange interaction and a nearest neighbor Fe-Rh FM exchange interaction. Thus, at $0 \mathrm{~K}$, the AFM phase gains its low energy from a dominating AFM exchange interaction which, with increasing volume-per-atom, reduces allowing the FM interaction between the $\mathrm{Fe}$ and $\mathrm{Rh}$ atoms to eventually dominate the energetics resulting in the FM phase becoming the ground state at a high enough (experimentally inaccessible) volume, despite the additional cost of the Rh atom having an induced magnetic moment.

Separate to the empirical model development within a Heisenberg Hamiltonian framework, Gruner et al ${ }^{27}$ have also developed an Ising model derived from their own extensive local DFT calculations. The authors argue that the use of an Ising model is justified because the FeRh phase transition occurs between two colinear magnetic configurations. Such a model does not admit spin-wave excitations and also is unable to describe the noncolinear variation of magnetic moment suggested by DFT work. ${ }^{23,25}$ Despite these restrictions, Monte Carlo simulations did reveal an AFM-FM transition at $322 \mathrm{~K}$ with a corresponding entropy change comparable to experiment. The model is unique in that it includes local volume fluctuations at the atomic scale.

The present work develops further an empirical Heisenberg model in which the moment magnitude of both the $\mathrm{Fe}$ and $\mathrm{Rh}$ atoms can vary via the addition of a Landau term. Thus both transverse and longitudinal moment fluctuations are considered on an equal footing. Doing so allows the model to be fitted directly to the earlier colinear DFT results of Moruzzi and Marcus $^{9}$ as a function of volume-per-atom, and to be compared reasonably well to the noncolinear DFT work of $\mathrm{Gu}$ and Antropov, ${ }^{23}$ and Sandratskii and Mavropoulos. ${ }^{25}$ The developed model considers only the relevant nearest neighbor exchange interaction between the $\mathrm{Fe}$ and $\mathrm{Fe}$, and the $\mathrm{Fe}$ and $\mathrm{Rh}$ moments. Within this framework, it is found that in order to stabilize the FM phase with respect to transverse moment fluctuations, a nearest-neighbor $\mathrm{Fe}-\mathrm{Fe}$ indirect-like exchange interaction is required. By exploiting an additional volumedependent nonmagnetic energy (equation-of-state) relation, the total cohesive energy of the system as a function of global 
volume-per-atom and moment degrees of freedom is obtained. This is, in turn, used in a Monte Carlo algorithm to model the finite-temperature properties of both the AFM and FM phases of the FeRh system. Despite the global volume restriction of the model, the finite-temperature simulations infer that local volume fluctuations play a crucial role in the magnetic and structural transition. This result will be discussed within the frame work of recent pump-probe experiments in which both the structural and magnetic evolution of the phase transition have been measured at the picosecond time scale.

\section{MODEL DEVELOPMENT}

\section{A. Classical Landau-Heisenberg Hamiltonian: initial considerations}

To develop an empirical model that allows for variations in both moment magnitude (longitudinal fluctuations) and direction (transverse fluctuations), two well-established theoretical frameworks are exploited.

To model the longitudinal fluctuations, an analogy to Landau mean field theory ${ }^{28}$ is used. This entails that the magnetic energy is approximated as a power series with respect to the moment magnitude: the so-called Landau function which, due to time-reversal symmetry, generally contains only even powers of the moment. For such an expansion there exist two important examples. In systems where there exists a spontaneous magnetization, such as in ferromagnetic BCC $\mathrm{Fe}$, the Landau function takes the form

$$
E(\mathbf{M})=A|\mathbf{M}|^{4}-B|\mathbf{M}|^{2} .
$$

Here both $A$ and $B$ are positive to give the symmetry-breaking magnetic solutions at a finite magnetic moment equal to $\sqrt{B /(2 A)}$. In this context Eq. (1) represents a realization of the Stoner model ${ }^{29}$ and is the leading-order contribution to the magnetic energy for an itinerant system where the electronic density of states (DOS) is peaked in the vicinity of the Fermi energy and the onsite inter-orbital exchange is sufficient to satisfy the Stoner criterion for ferromagnetism. Indeed, for Eq. (1), the Stoner criterion corresponds to the stability condition, $B /(2 A)>0$, which is obviously met when both $A$ and $B$ are positive.

On the other hand, if the Stoner criterion is not met, by $B$ being negative, the Landau function becomes

$$
E(\mathbf{M})=A|\mathbf{M}|^{4}-B|\mathbf{M}|^{2}=-B|\mathbf{M}|^{2},
$$

where $A$ is set to zero (when only the leading-order term is needed). This may be viewed as representing the leadingorder magnetic energy for a metallic system, where the only stable solution is the nonmagnetic one (at $\mathbf{M}=0$ ) in which the energy increases quadratically with respect to a finite moment magnitude. The Stoner criterion, now not being met, is due to a too low value of either the electronic DOS in the vicinity of the Fermi energy and/or the onsite interorbital exchange.

Whilst the Stoner model can describe well the phenomenon of itinerant magnetism for certain metals, the usage of the above Landau functions need not be directly linked to Stoner magnetism. This is also the case for the metallic regime of Eq. (2). The framework of Landau mean field theory is far more general than that of the Stoner model, as is readily demonstrated for FeRh.
For application to the FeRh system, a Landau function must be chosen for both the $\mathrm{Fe}$ and $\mathrm{Rh}$ atom types. Due to the relative robustness of the $\mathrm{Fe}$ moment magnitude with respect to volume and temperature, a Stoner-type form [Eq. (1)] is clearly justified. For the Rh atom, the work of Sandratskii and Mavropoulos $^{25}$ suggests a form similar to Eq. (2), however the authors provide strong $a b$ initio evidence that this is not indicative of the $\mathrm{Rh}$ atom being a simple metallic atom. Indeed, their work shows, that the DOS undergoes significant changes in hybridization between the AFM and FM magnetic configurations at $0 \mathrm{~K}$. It therefore might seem remarkable that such a simple energy functional is sufficient, but it is worth pointing out that integrated DOS quantities such as total energy and total moment are often insensitive to the fine details of an electronic DOS. ${ }^{30}$

For the present FeRh empirical model, the Landau function for the Fe atom is therefore written as

$$
E_{\mathrm{Fe}}\left(\mathbf{M}_{\mathrm{Fe}}\right)=A_{\mathrm{Fe}}\left|\mathbf{M}_{\mathrm{Fe}}\right|^{4}-B_{\mathrm{Fe}}\left|\mathbf{M}_{\mathrm{Fe}}\right|^{2},
$$

where both $A_{\mathrm{Fe}}$ and $B_{\mathrm{Fe}}$ are positive, and for the $\mathrm{Rh}$ atom as

$$
E_{\mathrm{Rh}}\left(\mathbf{M}_{\mathrm{Rh}}\right)=-B_{\mathrm{Rh}}\left|\mathbf{M}_{\mathrm{Rh}}\right|^{2},
$$

where $B_{\mathrm{Rh}}$ is negative.

To model transverse fluctuations in the magnetic moment a minimal Heisenberg exchange model is first considered, in which only the nearest-neighbor Fe-Rh and second-nearestneighbor $\mathrm{Fe}-\mathrm{Fe}$ interactions are included, each respectively being parameterized by the exchange coupling constants $J_{\mathrm{FeRh}}$ and $J_{\mathrm{FeFe}}$. The total energy of an $N$ atom system is therefore given by

$$
E=\sum_{i=1}^{N} E_{t_{i}}\left(\mathbf{M}_{i}\right)-\frac{1}{2} \sum_{i \neq j=1}^{N} J_{t_{i} t_{j}} \mathbf{M}_{i} \cdot \mathbf{M}_{j},
$$

where $t_{i}$, the atom type, will either be Fe or Rh. It is noted that since both transverse and longitudinal fluctuations are allowed, the exchange parameters are in units of energy per Bohr magneton squared, that is, the moment magnitudes are not absorbed into the exchange constants.

The Hamiltonian of Eq. (5) will be referred to as the LandauHeisenberg Hamiltonian. It is worth pointing out that there is no contradiction in using a Landau-Heisenberg model to study a first-order phase transition, since (as will be shortly shown) this transition is with respect to two stable and independent solutions of the Hamiltonian, Eq. (5). In fact within each stable solution, a second-order phase transition occurs in going to the paramagnetic phase, physics that is well described by the use of such a Landau-Heisenberg Hamiltonian.

To determine the zero Kelvin ground state antiferromagnetic structure and the excited state ferromagnetic structure, a conventional cell of $2 \times 2 \times 2$ unit cubic cells consisting of sixteen atoms is used. Although this is not the primitive cell, its use facilitates a simple visualization of the BCC lattice and its corresponding magnetic structures (see Fig. 1), where only one of the body center $\mathrm{Rh}$ atoms is shown of the conventional cell.

Under the assumption that (i) both the AFM and FM magnetic structures are colinear, and (ii) there exists two (FCC) sublattice magnetic moment magnitudes $M_{\mathrm{Fe} 1}$ and $M_{\mathrm{Fe} 2}$ for the Fe atoms [as in Figs. 1(a)] and (iii), the Rh atoms have the 
magnetic moment magnitude $M_{\mathrm{Rh}}$, the energy per conventional cell can be written as

$$
\begin{aligned}
E_{\text {Colinear }}= & 4\left(A_{\mathrm{Fe}} M_{\mathrm{Fe} 1}^{4}-B_{\mathrm{Fe}} M_{\mathrm{Fe} 1}^{2}\right)+4\left(A_{\mathrm{Fe}} M_{\mathrm{Fe} 2}^{4}-B_{\mathrm{Fe}} M_{\mathrm{Fe} 2}^{2}\right) \\
& -8 B_{\mathrm{Rh}} M_{\mathrm{Rh}}^{2}-24 J_{\mathrm{FeFe}} M_{\mathrm{Fe} 1} M_{\mathrm{Fe} 2} \\
& -32 J_{\mathrm{FeRh}}\left(M_{\mathrm{Fe} 1}+M_{\mathrm{Fe} 2}\right) M_{\mathrm{Rh}} .
\end{aligned}
$$

Eq. (6) has the required magnetic symmetry that, if $M_{\mathrm{Fe} 1}=$ $-M_{\mathrm{Fe} 2}$, the contribution due to $J_{\mathrm{FeRh}}$ vanishes resulting in $M_{\mathrm{Rh}}=0$ via independent minimization of Eq. (4).

By equating to zero each differential with respect to the three moment magnitudes, stationary solutions of the above may be found for $M_{\mathrm{Fe} 1}, M_{\mathrm{Fe} 2}$ and $M_{\mathrm{Rh}}$. Doing so results in the AFM ground state solution being,

$$
\begin{gathered}
M_{\mathrm{Fe} 1}= \pm \sqrt{\frac{B_{\mathrm{Fe}}-3 J_{\mathrm{FeFe}}}{2 A_{\mathrm{Fe}}}} \\
M_{\mathrm{Fe} 2}=\mp \sqrt{\frac{B_{\mathrm{Fe}}-3 J_{\mathrm{FeFe}}}{2 A_{\mathrm{Fe}}}} \\
M_{\mathrm{Rh}}=0,
\end{gathered}
$$

with the energy per atom equaling,

$$
E=-\frac{\left(B_{\mathrm{Fe}}-3 J_{\mathrm{FeFe}}\right)^{2}}{8 A_{\mathrm{Fe}}} .
$$

Whereas the FM solution has the form,

$$
\begin{aligned}
M_{\mathrm{Fe} 1(\mathrm{Fe} 2)} & = \pm \sqrt{\frac{B_{\mathrm{Fe}}+3 J_{\mathrm{FeFe}}-16 \frac{J_{\mathrm{FeRh}}^{2}}{B_{\mathrm{Rh}}}}{2 A_{\mathrm{Fe}}}} \\
M_{\mathrm{Rh}} & = \pm 4 J_{\mathrm{FeRh}} \sqrt{\frac{B_{\mathrm{Fe}}+3 J_{\mathrm{FeFe}}-16 \frac{J_{\mathrm{FeRh}}^{2}}{B_{\mathrm{Rh}}}}{2 A_{\mathrm{Fe}} B_{\mathrm{Rh}}^{2}}}
\end{aligned}
$$

with the energy per atom equaling,

$$
E=-\frac{\left(B_{\mathrm{Fe}}+3 J_{\mathrm{FeFe}}-16 \frac{J_{\mathrm{FeRh}}^{2}}{B_{\mathrm{Rh}}}\right)^{2}}{8 A_{\mathrm{Fe}}} .
$$

Inspection of Eq. (12) immediately reveals $J_{\mathrm{FeRh}}$ must be positive (FM) if the Rh moment is to be in the same direction as the FM Fe moments, and thus the exchange interaction between the nearest neighbor Fe and Rh atoms must be FM. The (always) higher energy AFMI soution discussed in Ref. 9 is also admitted by Eq. (6), and will not be considered further in this work.

In the work of Moruzzi and Marcus, ${ }^{9}$ DFT data as a function of volume-per-atom was calculated including the energy difference between the AFMII and FM magnetic states, the moment magnitude of the Fe atoms in the AFMII state, and the moment magnitudes of the $\mathrm{Fe}$ and $\mathrm{Rh}$ atoms in the FM state. Additionally, calculations were performed in which the total magnetic moment of the employed conventional cell was constrained to a fixed value from which the total energy relative to the AFMII ground-state energy and corresponding total $\mathrm{Fe} / \mathrm{Rh}$ moments were calculated for different values of the volume-per-atom. Such data is useful since it gives the energy barrier between the AFMII and FM magnetic structures when only the longitudinal moment component can be varied (that is, the colinearity of the moments is retained). In the present model, these quantities can also be investigated by obtaining the stable solutions of Eq. (6) with respect to the constraint $M_{\text {Total }}=4 M_{\mathrm{Fe} 1}+4 M_{\mathrm{Fe} 2}+8 M_{\mathrm{Rh}}$, via the Lagrange multiplier technique. In this way the stable solutions can be analytically obtained as a function of both the $M_{\text {Total }}$ and volume-per-atom. While such equations are analytically tractable, it becomes more efficient for the fitting to approximate the energy function at the AFMII and FM regions as two separate quadratic functions with respect to $M_{\text {Total }}$, the intersection of which will give a reasonable estimate of the colinear energy barrier between the AFMII and FM magnetic states. This approximation is found to work extremely well [see Figs. 3(c) and 3(d)].

The DFT data of Moruzzi and Marcus, ${ }^{9}$ will now be used to obtain numerical estimates of the Stoner $\left(A_{\mathrm{Fe}}, B_{\mathrm{Fe}}\right.$, and $\left.B_{\mathrm{Rh}}\right)$ and exchange parameters ( $J_{\mathrm{FeFe}}$ and $\left.J_{\mathrm{FeRh}}\right)$ of Eq. (6), as a function of volume-per-atom defined by a Wigner-Seitze radius $r_{\mathrm{WS}}$. To do this, these parameters are represented as a truncated power series

$$
\sum_{n=0}^{3} a_{n}\left(r_{\mathrm{WS}}-r_{\mathrm{WS}, 0}\right)^{n},
$$

about some chosen reference Wigner-Seitze radius $r_{\mathrm{WS}, 0}$. For the present work, a simulated annealing strategy ${ }^{31}$ is employed to find the optimal $\left\{a_{n}\right\}$ for each Stoner and each exchange parameter. Table I lists the corresponding values for the optimal fit.

Figure 2(a) plots the energy difference between the AFMII and FM magnetic states and Fig. 2(b) plots the magnitudes of the AFMII Fe moment, and the FM Fe and Rh moments as a function of volume-per-atom, along with the corresponding DFT data of Moruzzi and Marcus. ${ }^{9}$ Agreement between the optimal fit and DFT values is quite good. In the fit, the lowest few Wigner-Seitz radii data had a decreased weight such that the fit would be optimal for the typical experimental regime of atomic volumes. Figure 2(c) now plots the resulting absolute values of the magnetic energy for the two phases, demonstrating the model exhibits the known phenomenon that upon compression electronic correlations are reduced resulting in a decreasingly negative magnetic energy and a reduced magnetic moment [Fig. 2(b)] — the so-called magneto-volume effect. It is noted that the absolute values of the magnetic energy are not fitted.

Moruzzi and Marcus ${ }^{9}$ also obtained DFT derived equationof-state (EOS) curves for the AFMII and FM magnetic structures, giving them in terms of the four-parameter Morse function

$$
E\left(r_{\mathrm{WS}}\right)=A-2 D \exp ^{-\lambda\left(r_{\mathrm{WS}}-r_{0}\right)}+D \exp ^{-2 \lambda\left(r_{\mathrm{WS}}-r_{0}\right)} .
$$

To reproduce such EOS curves for both magnetic structures, an EOS curve must be obtained for the nonmagnetic contribution to the total energy: a volume-per-atom dependent energy contribution that is then added to the volume-per-atom dependent magnetic energies [Fig. 2(c)] to obtain the total energy. In the present work, the single nonmagnetic EOS curve [parameterized by Eq. (15)] is obtained by fitting it to the difference between the obtained absolute magnetic energies [Fig. 2(c)] and the corresponding magnetic EOS curves of Moruzzi and Marcus. It is noted that this stage 
TABLE I. Optimal coefficients of the truncated power series representation [Eq. (14)] for the Landau and Heisenberg exchange parameters, with $r_{\mathrm{WS}, 0}=2.878335833$ atomic units (au). Also listed are the optimal parameters for the nonmagnetic Morse representation of the equation of state. For modifications that stabilize the FM phase and improve its equilibrium energy, see Sec. II C.

\begin{tabular}{|c|c|c|c|c|}
\hline Parameter & $a_{0}(\mathrm{mRy})$ & $a_{1}(\mathrm{mRy} / \mathrm{au})$ & $a_{2}\left(\mathrm{mRy} / \mathrm{au}^{2}\right)$ & $a_{3}\left(\mathrm{mRy} / \mathrm{au}^{3}\right)$ \\
\hline$A_{\mathrm{Fe}}$ & 0.93368 & $-1.18306 \times 10^{-2}$ & 0.84856 & 2.18889 \\
\hline$B_{\mathrm{Fe}}$ & 17.95829 & 15.94231 & -15.83892 & 25.49692 \\
\hline$B_{\mathrm{Rh}}$ & -19.40221 & -34.35754 & 45.91000 & 99.52522 \\
\hline$J_{\mathrm{FeFe}}$ & -0.32865 & 0.39170 & -2.37376 & -5.68641 \\
\hline$J_{\mathrm{FeRh}}$ & 1.54646 & 1.93756 & -0.63683 & 9.09707 \\
\hline \multirow[t]{2}{*}{ Morse parameter } & $A_{\mathrm{NM}}(\mathrm{mRy})$ & $D_{\mathrm{NM}}(\mathrm{mRy})$ & $\lambda_{\mathrm{NM}}(1 / \mathrm{au})$ & $r_{\mathrm{WS}, \mathrm{NM}}(\mathrm{au})$ \\
\hline & 549.81581 & 512.00935 & 1.31446 & 2.72765 \\
\hline
\end{tabular}

of the fitting is performed after the fitting of the magnetic energies and moments. Indeed, since the absolute values of the magnetic energies are not known in the work of Moruzzi and Marcus, the final form of the nonmagnetic EOS curve is somewhat arbitrary, it being necessary for the calculation
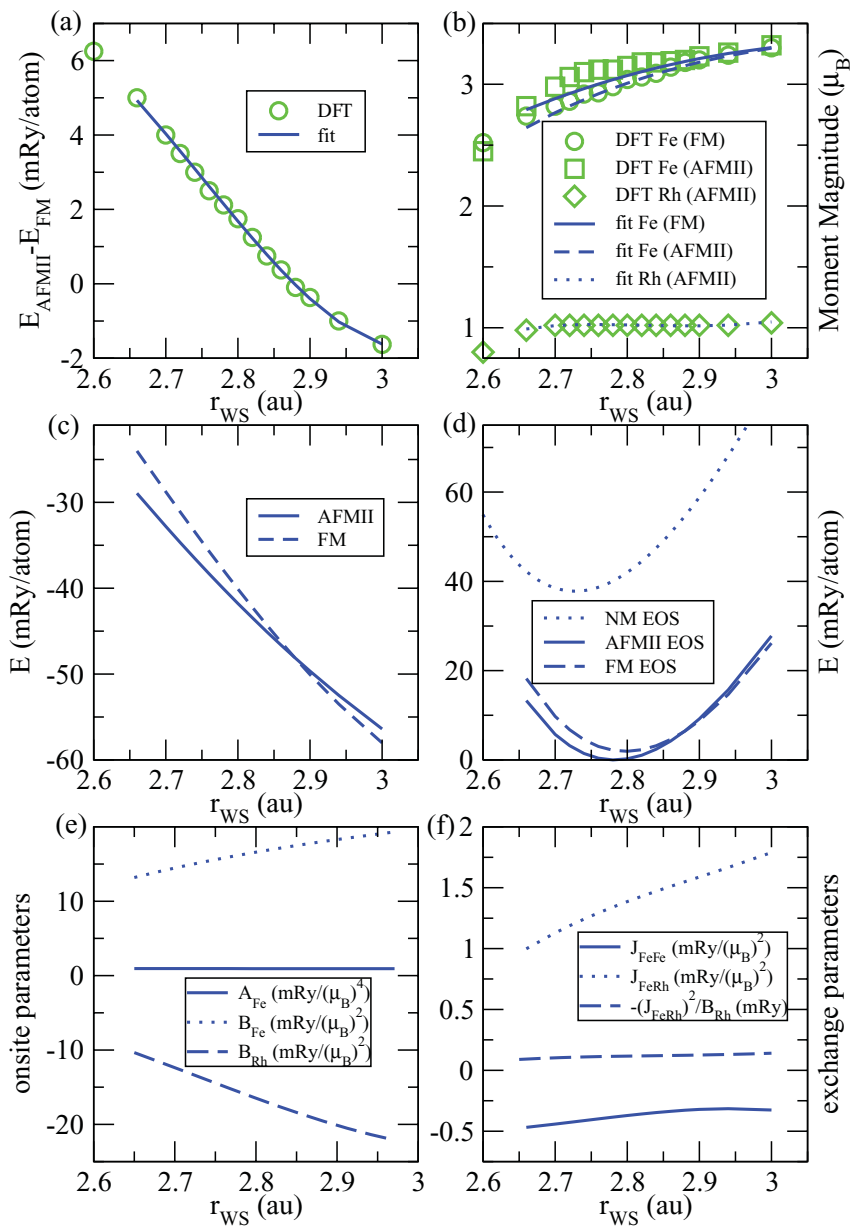

FIG. 2. (Color online) Comparison between optimal fit and DFT data as a function of Wigner-Seitz radius for (a) the magnetic energy difference between the AFMII and FM phases, and (b) the magnetic moments of both phases. (c) Absolute magnetic energies and (d) equation of state of the AFMII and FM phases as a function of Wigner-Seitz radius. (e) and (f) show the resulting Landau and Heisenberg exchange parameters as function of Wigner-Seitz radius. of the given EOS curves of the AFMII and FM phases, and (as will be shown in Sec. III) for the inclusion of volume fluctuations at finite temperature. Table I lists the associated optimal Morse parameters of Eq. (15), and Fig. 2(d) displays the resulting EOS curves for both magnetic phases and the nonmagnetic phase. The nonmagnetic curve compares quite well with those calculated by Gruner et $a l^{27}$ Figures 2(a), 2(c), and 2(d) all show the important feature that, at $0 \mathrm{~K}$, below a critical Wigner-Seitz radius the AFMII phase is the ground-state structure.

Figures 2(e) and 2(f) display the actual values of the Landau and Heisenberg exchange parameters as a function of Wigner-Seitz radius. In Fig. 2(e), all Landau parameters have the required sign, where $B_{\mathrm{Fe}}$ and $\left|B_{\mathrm{Rh}}\right|$ increase with increasing Wigner-Seitz radius, and $A_{\mathrm{Fe}}$ remains approximately constant with respect to volume. In Fig. 2(f), the exchange parameter between $\mathrm{Fe}$ and $\mathrm{Rh}$ is positive, indicating a FM interaction between the two atoms - a requirement for the Rh moment to be parallel to the Fe moments in the FM phase [Eq. (12)]. On the other hand, the optimal fit results in a AFM exchange interaction between the nearest neighbor Fe atoms. These results will be discussed in further detail in Sec. IV.

Figure 3 displays the predictions of the model as a function of total colinear moment $M_{\mathrm{Total}}=4 M_{\mathrm{Fe} 1}+4 M_{\mathrm{Fe} 2}+8 M_{\mathrm{Rh}}$. Here Figs. 3(a) and 3(b) plot the individual magnetic moments and Figs. 3(c) and 3(f), the magnetic energy, for two values of the Wigner-Seitz radius considered in Ref. 9. For a total moment of zero, the lower panels show the ground-state magnetic structure is that of the AFMII phase and by increasing the total moment the FM phase eventually becomes the ground state. The magnetic energy at which the ground state changes constitutes the relevant energy barrier between the two phases when only colinear variations of magnetic moment are considered. The corresponding moment variation as a function of total colinear moment confirm this interpretation. All curves in Fig. 3 compares extremely well to those obtained by Moruzzi and Marcus 9 (see their Figs. 4 and 5), where poorest agreement occurs in the regions far from the ground-state phases, an expected result, due to the simple fourth-degree polynomial form of the Landau-Heisenberg Hamiltonian.

\section{B. Noncolinear excitations}

The solutions found in the previous section are now investigated in terms of variations of the transverse degrees of 

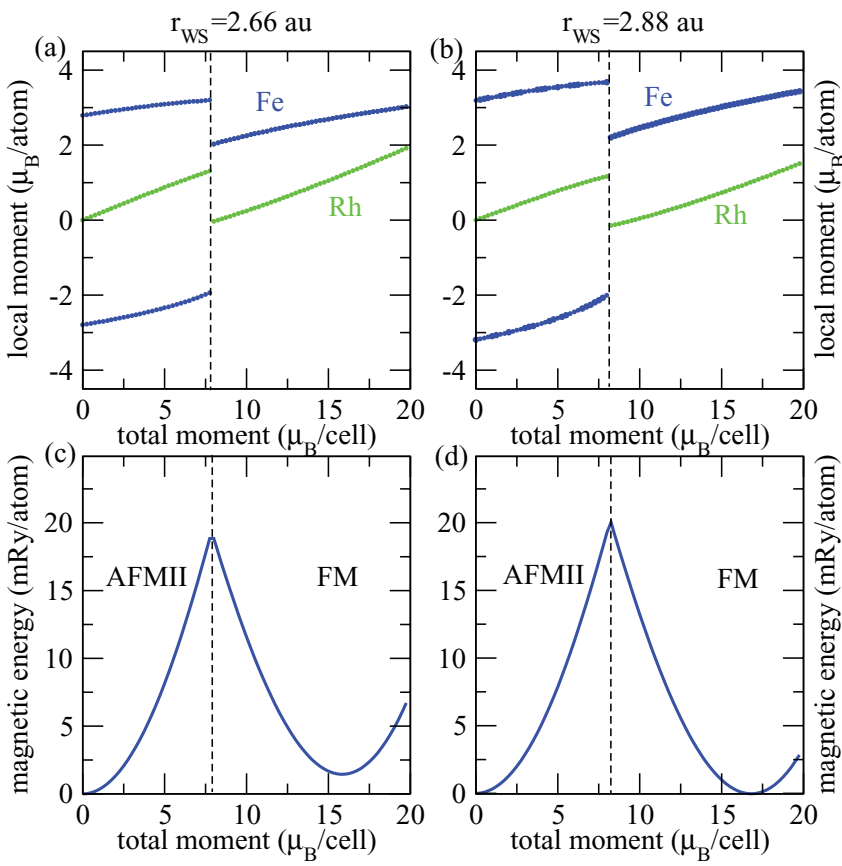

FIG. 3. (Color online) Ground state magnetic moments of the Fe and $\mathrm{Rh}$ atoms as a function of total magnetic moment per conventional cell for a Wigner-Seitz radius of (a) $2.66 \mathrm{au}$ and (b) $2.88 \mathrm{au}$. (c) and (d) show the corresponding ground-state magnetic energy dependencies.

freedom of the magnetic moments, which lead to noncolinear arrangements of the magnetic structure. To do this, a similar approach is taken to that of Sandratskii and Mavropoulos, ${ }^{25}$ where the focus was, in part, to extract information on the relevant exchange mechanisms from their DFT results.

The first such variation involves a rotation of the $\mathrm{Rh}$ moment in the FM magnetic state away from the Fe moment, keeping the direction of the Fe moments fixed. In analogy to the DFT approach, for each such angle of rotation, Eq. (5) is minimized with respect to the moment magnitudes to obtain the relevant stable solution. Figure 4(a) displays the resulting moment magnitudes and Fig. 4(b), the variation in magnetic energy as a function of angle for a range of volume-per-atom values. As a function of angle, the moment magnitude on the Fe atoms varies little whereas on the $\mathrm{Rh}$ atoms it decreases to zero when perpendicular to the $\mathrm{Fe}$ moment, resulting in a magnetic configuration that is unstable. The curves compare extremely well to those in Fig. 6 of Ref. 25. For all quantities, a family of curves are plotted that span the Wigner-Seitz radii from $2.78 \mathrm{au}$ (marked in red) to 2.9 au. Note that the present energy curves are plotted as energy per atom, whereas in Ref. 25 they are plotted as energy per fundamental unit (per one $\mathrm{Fe}$ and one $\mathrm{Rh}$ atom).

The second such variation begins with the AFMII state and ends with the FM state providing a candidate transition pathway between the two phases. This is achieved by canting the $\mathrm{Fe}$ moments, originally along, say, the [100] direction toward the [010] direction with the Rh moment constrained to be along this [010] direction. Within these constraints the energy is minimized to find the stable moment magnitudes of the Fe and $\mathrm{Rh}$ atoms. Figure 4(c) displays the resulting moment magnitudes and Fig. 4(d) the variation in magnetic energy as
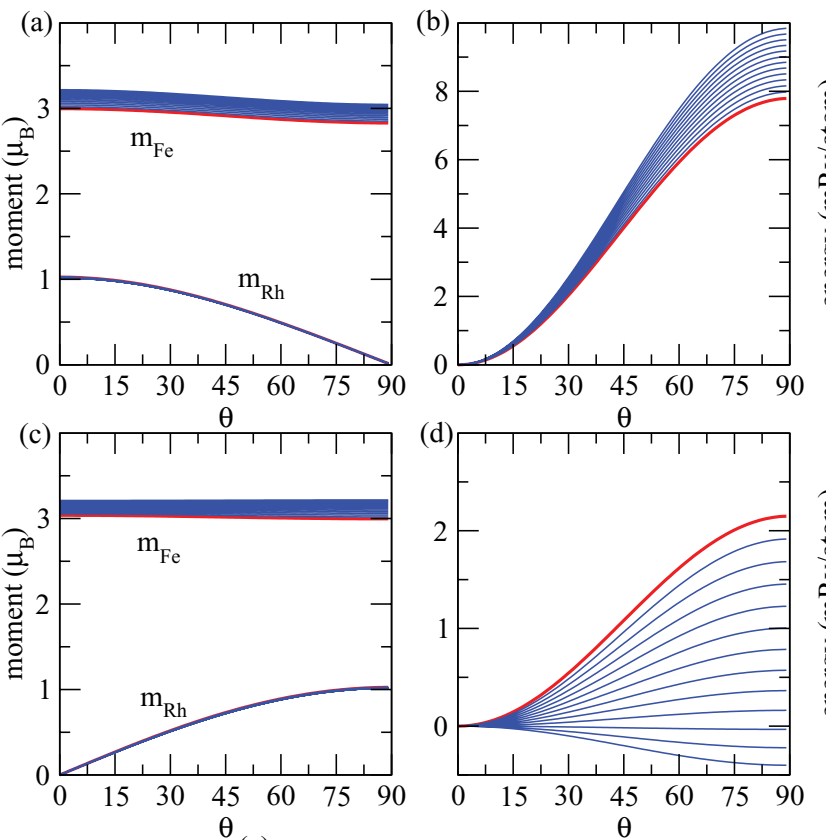

\section{ฮ્ટ}

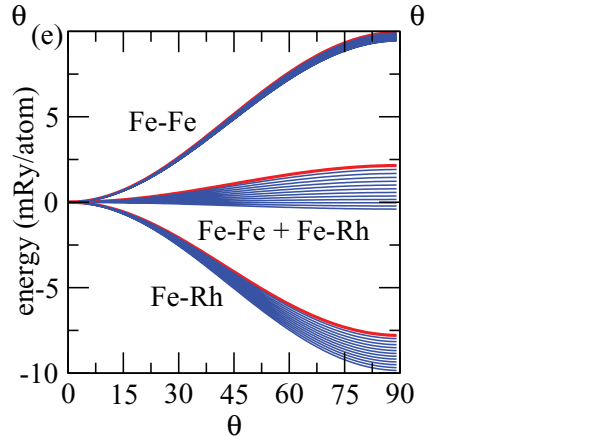

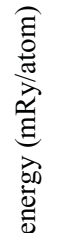

FIG. 4. (Color online) Noncolinear dependency of the Fe and $\mathrm{Rh}$ moments and their corresponding magnetic energy variation. (a) and (b) represent a variation of the Rh moment direction in the FM phase with the Fe moment direction fixed, and (c) and (d) the variation in which the Fe moments of the AFMII phase are canted towards an orthogonal Cartesian direction, with the Rh moment being constrained to point along that direction. This latter variation constitutes a magnetic structural excitation linking the AFMII and FM phases. (e) Represent a similar canting variation with the Rh moment constrained to be zero, thus directly probing the $\mathrm{Fe}-\mathrm{Fe}$ exchange interaction. For all quantities, a family of curves are plotted that span the Wigner-Seitz radii from 2.78 (marked in red) to $2.9 \mathrm{au}$.

a function of angle for a range of volume-per-atom values. Inspection of Fig. 4(d) reveals that when the FM phase has an energy greater than the AFMII phase (at $\theta=90^{\circ}$ ) the former is unstable with respect to $\theta$. Although DFT demonstrates this for low Wigner-Seitz radii, at higher radii the FM phase stabilizes (see Fig. 7 in Ref. 25). Thus the model developed in the previous section is unable to describe a crucial feature of the system where the higher-energy FM phase, at large enough Wigner-Seitz radii, exists at a local energy minimum. The resolution of this deficiency will be presented in the preceding section.

The third noncolinear moment variation carried out by Sandratskii and Mavropoulos ${ }^{25}$ involves a similar canting of the Fe moments from the AFMII phase, however, now with the moment magnitude of the Rh being set identically 


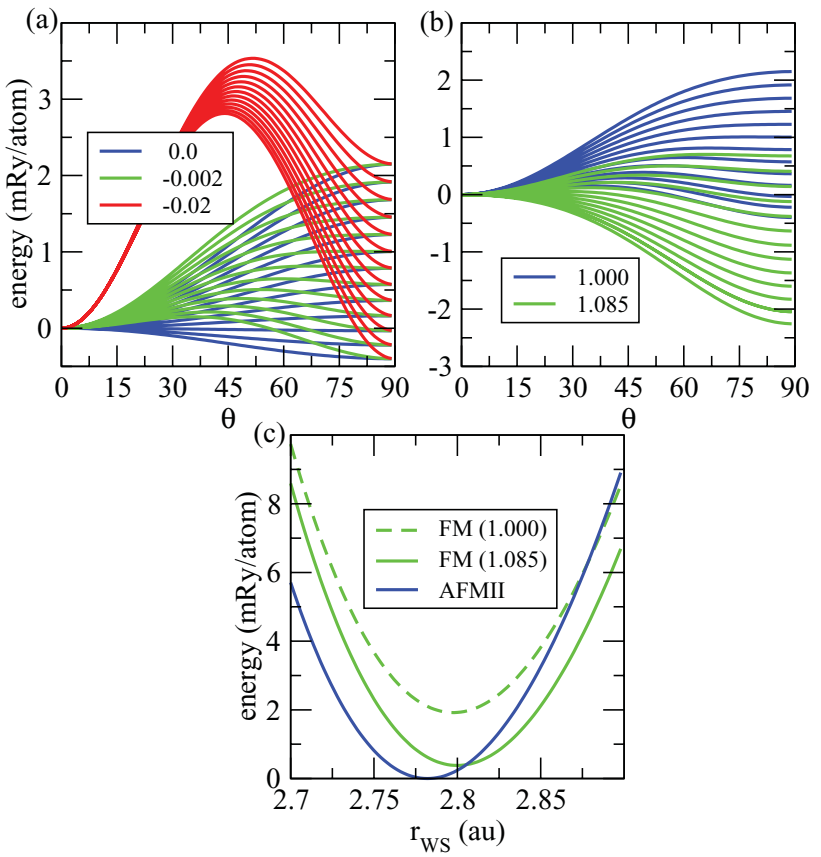

FIG. 5. (Color online) Variation of the magnetic energy difference between the AFMII and FM phases [see Fig. 4(d)] for different values of (a) $J_{\mathrm{FeFe}}^{(2)}$ and (b) fractional change in $J_{\mathrm{FeRh}}$ (with $J_{\mathrm{FeFe}}^{(2)}=-0.002$ ). In both (a) and (b) the uppermost curve of each family of curves represents a Wigner-Seitz radius of 2.70 au and the lowermost curve a radius of 2.9 au. (c) Displays the corresponding equation of state curves for the AFMII and FM phases with $J_{\mathrm{FeFe}}^{(2)}=-0.002$ and $1.085 J_{\mathrm{FeRh}}$.

to zero. It is argued this variation directly probes the $\mathrm{Fe}-\mathrm{Fe}$ exchange interaction. The corresponding energy curves are plotted in Fig. 4(e) and labeled as the Fe-Fe curves. Following Ref. 25, this figure also replots those of Fig. 4(d), labeling them as $\mathrm{Fe}-\mathrm{Fe}+\mathrm{Fe}-\mathrm{Rh}$, since this variation probes both the FeFe and FeRh exchange interaction. The difference of these curves is also plotted and labeled as Fe-Rh with the interpretation that this represents predominantly the exchange interaction between the $\mathrm{Fe}$ and $\mathrm{Rh}$ moments. Comparison with the DFT work of Sandratskii and Mavropoulos ${ }^{25}$ reveals quite good agreement, however in the DFT work this energy curve depends only weakly on volume whereas in the present fit there exists a clear non-negligible volume dependence. The latter is also reflected in Fig. 2(f) which indeed shows an explicit dependence of $J_{\mathrm{FeRh}}$ on volume, a quantity which Ref. 25 states is robust against volume-per-atom changes. The optimal fitting procedure undertaken in Sec. II A therefore appears not to enforce this feature. It however should be noted that in all stationary energy expressions arising from Eq. (5), the Fe-Rh contribution takes the form, $J_{\mathrm{FeRh}}^{2} / B_{\mathrm{Rh}}$, which when plotted [see Fig. 2(f)] is found to be robust against volume changes.

\section{The final model}

To stabilize the FM phase the Hamiltonian presented in Eq. (5) must be modified. To facilitate an energy barrier between the AFMII and FM phases, inspection of Fig. 4(d) suggests an effective angular dependence between the second nearest neighbor Fe moments that needs to be more rapid than
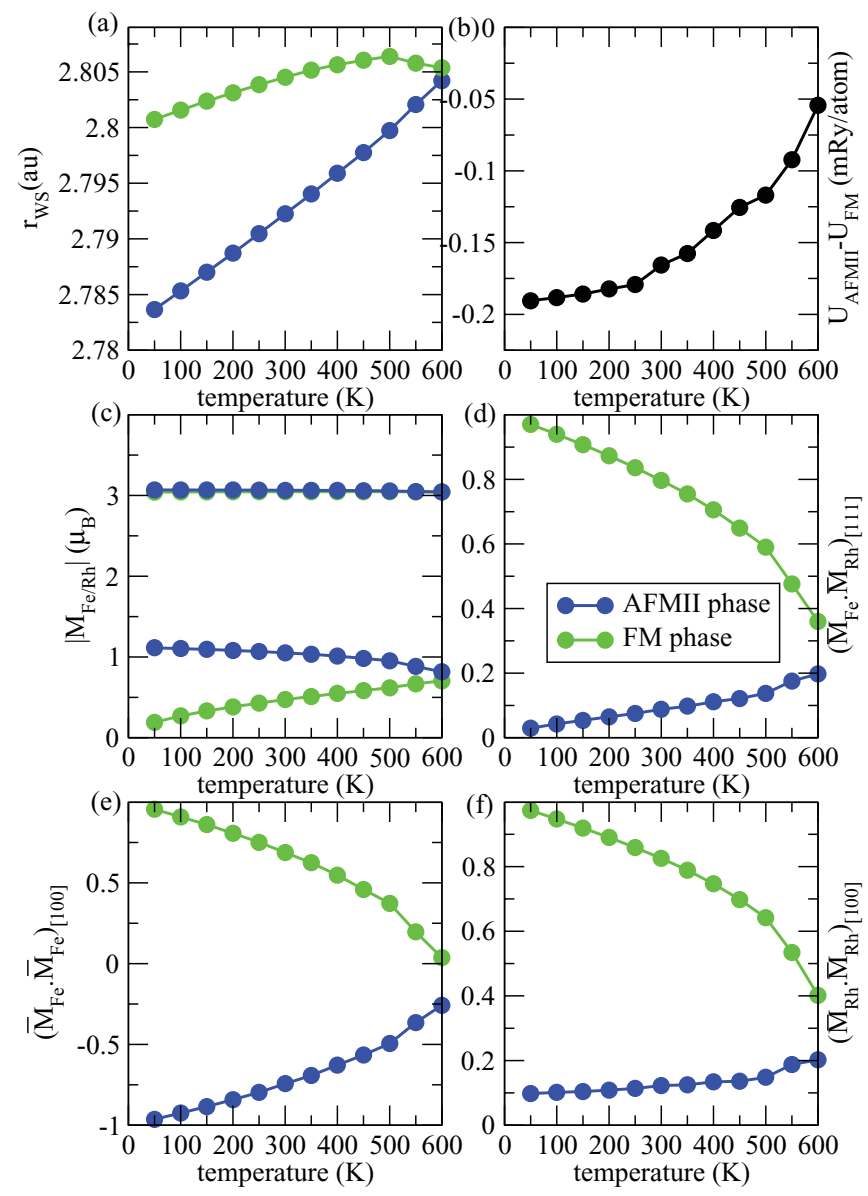

FIG. 6. (Color online) Average thermal properties as a function of temperature derived from a Monte Carlo simulation. The figures show data for separate simulations of the AFMII and FM magnetic structures, for (a) volume-per-atom measured as the Wigner-Seitz radius, (b) the internal energy difference between the AFMII and FM magnetic structure, (c) the moment magnitudes for the $\mathrm{Fe}$ and $\mathrm{Rh}$ atoms, and the normalized intersite correlation functions between the nearest neighbor (d) Fe and Rh moments, (e) the Fe and Fe moments, and (f) the Rh and Rh moments. In (c), the Fe magnetic moment magnitudes of the AFMII and FM phase are indistinguishable at the scale of the graph.

the cosine dependence explicitly given by the $\mathrm{Fe}-\mathrm{Fe}$ exchange interaction. The canting of the Fe AFM moments towards an orthogonal Cartesian direction and the corresponding emergence of a Rh moment along that direction, suggests that introducing more distant exchange interactions will not suffice. The simplest additional interaction that gives the required barrier, and also preserves the $O(3)$ symmetry class of the Heisenberg model, is a nearest neighbor interaction between the Fe and Rh that scales as the cosine squared. Specifically, the interaction term will have the exchange form

$$
-J_{\mathrm{FeFe}}^{(2)}\left(\mathbf{M}_{\mathrm{Fe} 1} \cdot \mathbf{M}_{\mathrm{Fe} 2}\right)^{2} .
$$

In what follows, this term will be referred to as quadratic exchange. Such a term, when added to the model, requires no refit to the parametrization derived from the colinear DFT in Sec. II A. Indeed, when including the interaction arising from Eq. (16), the colinear energy [analogous to Eq. (6)] 

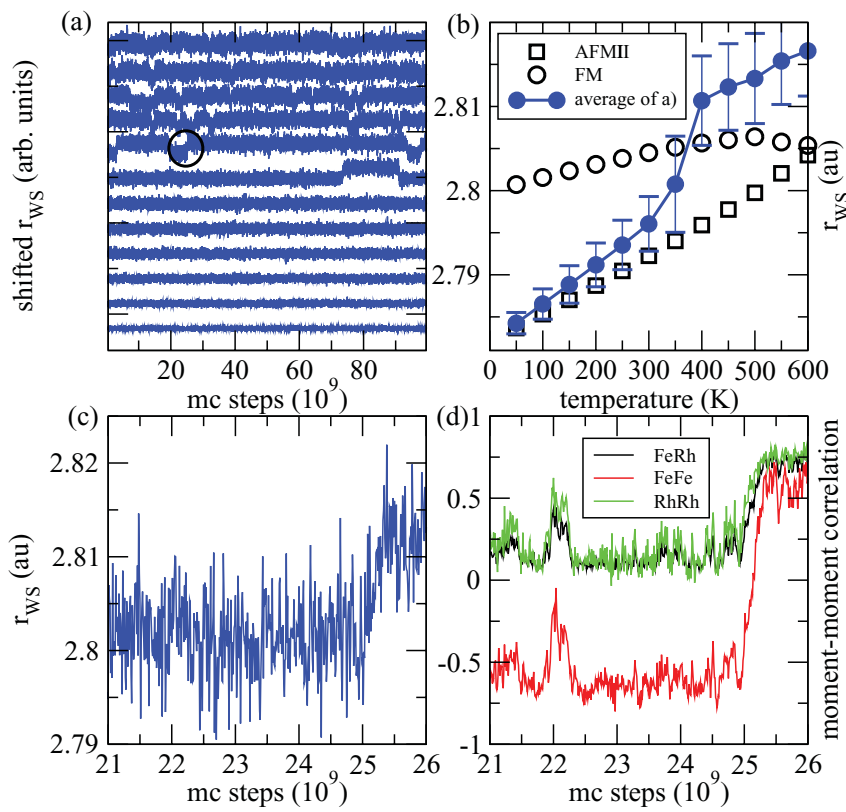

FIG. 7. (Color online) Results of MC simulations as a function of temperature. Here (a) displays the MC evolution of the Wigner-Seitz radius for each temperature, where the lowest curve corresponds to the $50 \mathrm{~K}$ MC simulation and the highest to the $600 \mathrm{~K}$ simulation. The radii for the greater than $50 \mathrm{~K} \mathrm{MC}$ simulations have been progressively shifted upward. (b) Displays the average Wigner-Seitz radius as a function of temperature. The equilibrium radii of Fig. 6(a) are also shown in this figure. (c) Shows a closeup of the Wigner-Seitz radius evolution of the $400 \mathrm{~K}$ data for the circled region in (b) and (d) shows the corresponding intersite correlation functions for the FeRh, FeFe, and RhRh nearest neighbor moments.

becomes,

$$
\begin{aligned}
E_{\mathrm{Colinear}}= & 4\left(A_{\mathrm{Fe}} M_{\mathrm{Fe} 1}^{4}-B_{\mathrm{Fe}} M_{\mathrm{Fe} 1}^{2}\right)+4\left(A_{\mathrm{Fe}} M_{\mathrm{Fe} 2}^{4}-B_{\mathrm{Fe}} M_{\mathrm{Fe} 2}^{2}\right) \\
& -8 B_{\mathrm{Rh}} M_{\mathrm{Rh}}^{2}-24 J_{\mathrm{FeFe}} M_{\mathrm{Fe} 1} M_{\mathrm{Fe} 2} \\
& -24 J_{\mathrm{FeFe}}^{(2)} M_{\mathrm{Fe} 1}^{2} M_{\mathrm{Fe} 2}^{2}-32 J_{\mathrm{FeRh}}\left(M_{\mathrm{Fe} 1}+M_{\mathrm{Fe} 2}\right) M_{\mathrm{Rh}},
\end{aligned}
$$

resulting in an additional biquadratic term with respect to the two Fe moment magnitudes. Because of this biquadratic term, the AFMII magnetic energy expression takes the form

$$
E=-\frac{\left(B_{\mathrm{Fe}}-3 J_{\mathrm{FeFe}}\right)^{2}}{8\left(A_{\mathrm{Fe}}-3 J_{\mathrm{FeFe}}^{(2)}\right)},
$$

and the FM magnetic energy,

$$
E=-\frac{\left(B_{\mathrm{Fe}}+3 J_{\mathrm{FeFe}}-\frac{16 J_{\mathrm{FeRh}}^{2}}{B_{\mathrm{Rh}}}\right)^{2}}{8\left(A_{\mathrm{Fe}}-3 J_{\mathrm{FeFe}}^{(2)}\right)} .
$$

Equations (18) and (19) are of similar form to Eqs. (10) and (13), and can be derived from them via the substitution, $A_{\mathrm{Fe}} \rightarrow$ $A_{\mathrm{Fe}}-3 J_{\mathrm{FeFe}}^{(2)}$. This is also the case for the corresponding moments. Thus in the colinear geometry the addition of the quadratic-exchange term renormalizes the $A_{\mathrm{Fe}}$. This simple relationship entails that, upon giving a value to $J_{\mathrm{FeFe}}^{(2)}, A_{\mathrm{Fe}}$ can be shifted by $3 J_{\mathrm{FeFe}}^{(2)}$ to retain the parametrization of Sec. II A. Figure 5(a) replots the noncolinear variation associated with
Fig. 4(d), and also for two nonzero values of $J_{\mathrm{FeFe}}^{(2)}$, demonstrating that quadratic exchange directly gives an energy barrier of any reasonable value for small values of the quadraticexchange parameter $\left(-0.02\right.$ and $\left.-0.002 \mathrm{mRy} / \mu_{\mathrm{B}}^{4}\right)$ with the magnetic energies of the AFMII and FM phases unchanged. The addition of such an interaction affects negligibly all other quantities shown in Fig. 4. For simplicity The present model will assume that the parameter $J_{\mathrm{FeFe}}^{(2)}$ is a constant independent of volume-per-atom.

As discussed in Sec. I, the works of Moruzzi and Marcus ${ }^{9}$ and Sandratskii and Mavropoulos ${ }^{25}$ both employed a local density approximation for the exchange functional. On the other hand, $\mathrm{Gu}$ and Antropov ${ }^{23}$ used a nonlocal exchange functional and found that the energy difference of the AFMII and FM equilibrium phases was reduced from $1.89 \mathrm{mRy} /$ atom to $0.206 \mathrm{mRy} /$ atom, the latter of which is more agreeable to a known experimental value of $0.196 \mathrm{mRy} /$ atom. $^{24}$ This is a considerable energy reduction, and is expected to strongly affect the nature of the transition between these two phases at finite temperature. It is therefore desirable to modify the obtained optimization to reproduce this feature, again without performing a refit.

Inspection of Eqs. (10) and (13) show that, at $T=0$, the energy of the AFMII phase does not depend on parameters involving the $\mathrm{Rh}$ atoms (since its moment is zero) and that the energy of the FM phase will depend, in part, on the ratio $J_{\mathrm{FeRh}}^{2} / B_{\mathrm{Rh}}$. Therefore modifying $J_{\mathrm{FeRh}}$ and/or $B_{\mathrm{Rh}}$ will change the energy of only the FM phase. Since transverse moment fluctuations are believed to play a critical role, the present work chooses to modify $J_{\mathrm{FeRh}}$. Figure $5(\mathrm{~b})$ displays similar energy curves such as Figs. 4(d) and 5(a), for a value of $J_{\mathrm{FeFe}}^{(2)}=-0.002 \mathrm{mRy} / \mu_{\mathrm{B}}^{4}$ demonstrating the effect of changing $J_{\mathrm{FeRh}}$ by a factor of 1.085 . The curve does indeed show that the energy difference between the two equilibrium magnetic phases is greatly affected by only a slight variation in the Fe-Rh exchange parameter. The resulting EOS curves shown in Fig. 5(c) compare well to that of the nonlocal DFT work of $\mathrm{Gu}$ and Antropov ${ }^{23}$ [see their Fig. 1(a)]. Again such a modification affects in a negligible way the other properties of the model. As evidenced by the present EOS curves, the developed model underestimates the absolute value of the equilibrium atomic volumes when compared to the nonlocal DFT work. To improve the absolute equilibrium volume per atom values would entail a refit, a task that is not performed in the present work.

Thus the final model constitutes the parametrization given in Table I with $J_{\mathrm{FeFe}}^{(2)}=-0.002 \mathrm{mRy} / \mu_{\mathrm{B}}^{4}$ and the $J_{\mathrm{FeRh}}$ parameter modified by a factor of 1.085 . It is noted that these two optimal parameters were obtained by hand.

\section{FINITE TEMPERATURE PROPERTIES}

\section{A. Free energy, pressure, and thermal expansion: a Monte Carlo simulation strategy}

The energy per atom as a function volume-per-atom, $\Omega$, and a given magnetic moment configuration may be now written as a sum of a nonmagnetic and magnetic contribution

$$
E\left(\Omega ;\left\{\mathbf{M}_{i}\right\}\right)=E_{\mathrm{NM}}(\Omega)+E_{\mathrm{M}}\left(\Omega ;\left\{\mathbf{M}_{i}\right\}\right)
$$


where the nonmagnetic term is the EOS function and the magnetic term is the developed Heisenberg-Landau Hamiltonian. At fixed volume per atom, $\Omega$, the free energy with respect to only the magnetic degrees of freedom is given by

$$
\begin{aligned}
F(\Omega, T) & =E_{\mathrm{NM}}(\Omega)-k_{\mathrm{B}} T \ln Z_{\mathrm{M}}(\Omega, T) \\
& =E_{\mathrm{NM}}(\Omega)+F_{\mathrm{M}}(\Omega, T),
\end{aligned}
$$

where $Z_{\mathrm{M}}(\Omega, T)$ is the partition function for the magnetic degrees of freedom and $F_{\mathrm{M}}(\Omega, T)$ is the corresponding magnetic free energy.

In the above, a finite temperature contribution arising from the lattice degrees of freedom is lacking. Such a contribution could be systematically added by constructing the nonmagnetic contribution from a pairwise or many-body interatomic force model, from which the dynamical matrix could be constructed [which would also contain a contribution from the $0 \mathrm{~K}$ magnetic energy in Eq. (20)] to obtain the vibrational frequencies $\left\{\omega_{i}, i=1,3 N\right\}$. This would give an extra term to the free energy, resulting in,

$F(\Omega, T)=E_{\mathrm{NM}}(\Omega)-k_{\mathrm{B}} T \sum_{i=1}^{3 N} \ln \left[\frac{\omega_{i}}{k_{\mathrm{B}} T}\right]+F_{\mathrm{M}}(\Omega, T)$,

where the middle term can be written as $F_{\text {Phonons }}(\Omega, T)$.

Presently the goal is to obtain the pressure of the system at a given volume-per-atom and temperature. Formally this is given by

$$
P(\Omega, T)=-\frac{d F(\Omega, T)}{d \Omega},
$$

which evaluates to

$$
\begin{aligned}
P(\Omega, T)= & -\frac{d E_{\mathrm{NM}}(\Omega)}{d \Omega}-\frac{3 k_{\mathrm{B}} T}{\Omega} \times \frac{1}{3 N} \sum_{i=1}^{3 N} \frac{\Omega}{\omega_{i}} \frac{d \omega_{i}}{d \Omega} \\
& -\left\langle\frac{d E_{\mathrm{M}}(\Omega)}{d \Omega}\right\rangle_{T} .
\end{aligned}
$$

The last term in the above equation is an ensemble average of the volume derivative of the magnetic energy with respect to the magnetic energy degrees of freedom and was obtained via the identity

$$
\frac{d F}{d \Omega}=-k_{\mathrm{B}} T \frac{d \ln Z}{d \Omega}=-\frac{k_{\mathrm{B}} T}{Z} \frac{d Z}{d \Omega}=\left\langle\frac{d E}{d \Omega}\right\rangle_{T} .
$$

The lattice degrees of freedom contribution contains the term $\Omega / \omega_{i} d \omega_{i} / d \Omega=\gamma_{i}$, which is the Grüneisen parameter for the eigenmode $i$. The summation over all modes may therefore be replaced with the average Grüneisen parameter, $\gamma$, giving

$$
P(\Omega, T)=-\frac{d E_{\mathrm{NM}}(\Omega)}{d \Omega}-\frac{3 k_{\mathrm{B}} T \gamma}{\Omega}-\left\langle\frac{d E_{\mathrm{M}}(\Omega)}{d \Omega}\right\rangle_{T} .
$$

The pressure arising from the magnetic contribution is given as a function of the relevant on-site and intersite moment correlation functions

$$
\begin{aligned}
\left\langle\frac{d E_{\mathrm{M}}(\Omega, T)}{d \Omega}\right\rangle_{T}= & \frac{1}{2}\left(\frac{d A_{\mathrm{Fe}}(\Omega)}{d \Omega}\left\langle M_{\mathrm{Fe}}^{4}\right\rangle_{M}\right. \\
& -\frac{d B_{\mathrm{Fe}}(\Omega)}{d \Omega}\left\langle M_{\mathrm{Fe}}^{2}\right\rangle_{M}-\frac{d B_{\mathrm{Rh}}(\Omega)}{d \Omega}\left\langle M_{\mathrm{Rh}}^{2}\right\rangle_{M}
\end{aligned}
$$

$$
\begin{gathered}
-3 \frac{d J_{\mathrm{FeFe}}(\Omega)}{d \Omega}\left\langle\mathbf{M}_{\mathrm{Fe}} \cdot \mathbf{M}_{\mathrm{Fe}}\right\rangle_{M} \\
-3 \frac{d J_{\mathrm{FeFe}}^{(2)}(\Omega)}{d \Omega}\left\langle\left(\mathbf{M}_{\mathrm{Fe}} \cdot \mathbf{M}_{\mathrm{Fe}}\right)^{2}\right\rangle_{M} \\
\left.-4 \frac{d J_{\mathrm{FeRh}}(\Omega)}{d \Omega}\left\langle\mathbf{M}_{\mathrm{Fe}} \cdot \mathbf{M}_{\mathrm{Rh}}\right\rangle_{M}\right) .
\end{gathered}
$$

Setting, $P(\Omega, T)=0$, defines a function $\Omega(T)$ giving the thermal expansion properties of the system.

The above results suggest a Monte Carlo approach to model the statistical mechanics with respect to a globally fluctuating volume-per-atom and local moment degrees of freedom, in which the change in energy of the Monte Carlo step can be

(i) a variation of a randomly selected moment. Since there is no restriction on the moment, this may be performed by sampling a spherically symmetric distribution of $\delta \mathbf{M}$ vectors where $|\delta \mathbf{M}| /|\mathbf{M}| \sim 0.001$ (say) with which the change in magnetic energy is calculated for a given global volume-peratom.

(ii) a variation of the global volume-per-atom $\Omega \rightarrow$ $\Omega+\delta \Omega$.

Here, for each Monte Carlo iteration, step (i) is performed with probability $N /(N+1)$ and step (ii) is performed with probability $1 /(N+1)$ to ensure a Markovian process. The change in energy required for step 2 is given by

$$
\delta E_{\mathrm{NM}}(\Omega)+\delta F_{\text {Phonons }}(\Omega, T)+\delta E_{\mathrm{M}}\left(\Omega ;\left\{\mathbf{M}_{i}\right\}\right),
$$

in which

$$
\begin{aligned}
\delta E_{\mathrm{NM}}(\Omega) & =E_{\mathrm{NM}}(\Omega+\delta \Omega)-E_{\mathrm{NM}}(\Omega) \\
\delta F_{\text {Phonons }}(\Omega, T) & =-3 k_{\mathrm{B}} T \frac{\delta \Omega}{\Omega} \frac{1}{3 N} \sum_{i=1}^{3 N} \frac{\Omega}{\omega_{i}} \frac{\delta \omega_{i}}{\delta \Omega} \\
& =-3 k_{\mathrm{B}} T \frac{\delta \Omega}{\Omega} \gamma \\
\delta E_{\mathrm{M}}\left(\Omega ;\left\{\mathbf{M}_{i}\right\}\right) & =E_{\mathrm{M}}\left(\Omega+\delta \Omega ;\left\{\mathbf{M}_{i}\right\}\right)-E_{\mathrm{M}}\left(\Omega ;\left\{\mathbf{M}_{i}\right\}\right) .
\end{aligned}
$$

\section{B. Finite-temperature Monte Carlo simulations}

Using the Monte Carlo (MC) approach developed in the previous section, the thermal properties of a many-moment FeRh system are investigated using the Landau-Heisenberg Hamiltonian with the parameters of Table I, and the appropriate modifications associated with the quadratic exchange term $\left(J_{\mathrm{FeFe}}^{(2)}=0.002 ; A_{\mathrm{Fe}} \rightarrow A_{\mathrm{Fe}}+3 J_{\mathrm{FeFe}}^{(2)}\right)$ and modified Fe-Rh exchange term $\left(J_{\mathrm{FeRh}} \rightarrow 1.085 J_{\mathrm{FeRh}}\right)$. The employed simulation cell contains $10 \times 10 \times 10$ unit cells giving a total of 2000 moments. The two relevant magnetic structures, AFMII and FM are considered in separate simulations, where each involves $30 \times 10^{9} \mathrm{MC}$ steps. For the Gruneisen parameter, the values given by Moruzzi and Marcus ${ }^{9}$ for the two phases are used: $\gamma=\gamma_{\text {AFMII }}=1.752$ for the AFMII phase simulations and $\gamma=\gamma_{\mathrm{FM}}=1.587$ for the FM phase simulations.

Figure 6 displays a number of observables as a function of temperature, where for each temperature, an average of the associated quantity is obtained after initial thermalization. Figure 6(a) displays the Wigner-Seitz radius as a function of temperature and it is seen that for both magnetic structures, the volume-per-atom increases with increasing temperature. Indeed for the lower temperature range of $50 \mathrm{~K}$ to $300 \mathrm{~K}$ the 
thermal expansion is quite linear allowing for the extraction of the relevant thermal expansion parameters. Doing so gives the linear coefficients for thermal expansion as $\alpha_{\mathrm{AFM}}=$ $1.2 \times 10^{-5} \mathrm{~K}^{-1}$ and $\alpha_{\mathrm{FM}}=5.4 \times 10^{-6} \mathrm{~K}^{-1}$. These numbers compare favourably with experimental thermal-expansion measurements, ${ }^{32}$ which give $\alpha_{T<T_{\mathrm{AFM}-\mathrm{FM}}}=0.95 \times 10^{-5} \mathrm{~K}^{-1}$ and $\alpha_{T>T_{\mathrm{AFM}-\mathrm{FM}}}=6 \times 10^{-6} \mathrm{~K}^{-1}$. For the FM phase, the volume-per-atom peaks and eventually decreases indicating strong anharmonic effects, which probably arise from the moment degrees of freedom, via Eq. (24). At the highest temperature considered, the phases have approximately the same volume-per-atom.

Figure 6(b) displays the internal energy difference between the two phases as a function of temperature. At the lowest temperatures, the thermal energy difference differs little from the $0 \mathrm{~K}$ value of approximately $-0.2 \mathrm{mRy}$. With increasing temperature the internal energy difference increases to -0.15 $\mathrm{mRy}$ at the known transition temperature regime (at approximately $300 \mathrm{~K}$ ) and to $-0.05 \mathrm{mRy}$ at $600 \mathrm{~K}$. Figure 6(c) now displays the magnetic moment magnitude for both the $\mathrm{Fe}$ and $\mathrm{Rh}$ atoms for both magnetic structures. As expected, the moment magnitude for the Fe atoms varies little with respect to temperature, due to its weak dependence on volume-per-atom. For the AFMII phase, the moment magnitude of the Rh atom steadily increases from its zero value at $0 \mathrm{~K}$, whereas for the FM phase it decreases. At the highest temperature of 600 $\mathrm{K}$ the $\mathrm{Rh}$ moment magnitudes of both magnetic phases are approximately the same.

Figures 6(d)-6(f) display the nearest-neighbor Fe-Fe, Fe$\mathrm{Rh}$, and $\mathrm{Rh}-\mathrm{Rh}$ moment-moment correlation functions for both magnetic phases. At low temperatures their inspection reveals clear AFM and FM structures. For the FM phase, all correlation functions decrease with temperature, indicating an eventual transition to the paramagnetic phase and a Curie temperature somewhere between $600 \mathrm{~K}$ and $700 \mathrm{~K}$. In the comparable finite-temperature MC simulations of Sandratskii and Mavropoulos, ${ }^{25}$ a Curie temperature of $600 \mathrm{~K}$ is found that, like the present work, agrees reasonably well with the experimental Curie temperature of $670 \mathrm{~K}^{3}$

For the case of the AFMII structure, Fig. 6(e) reveals a decrease of the Fe-Fe AFM correlation with increasing temperature suggesting a transition to the paramagnetic phase, at a Neel temperature that is not so different from that of the Curie temperature of the FM phase. In conjunction with this, increased correlation involving the $\mathrm{Rh}$ atom is observed with increasing temperature, suggesting a more complex transition to paramagnetism for the AFM structure. That both phases tend to the paramagnetic phase is consistent with the high-temperature convergence of the volume-per-atom data [Fig. 6(a)], the internal energy difference between the magnetic phases [Fig. 6(b)] and the Rh moment magnitudes [Fig. 6(c)].

The induced Rh moment seen in Fig. 6(c) for the AFMII phase has also been observed in the finite-temperature $\mathrm{MC}$ simulations of Sandratskii and Mavropoulos, ${ }^{25}$ who find that it plays an important role in determining the Neel temperature. Figure 6(d) shows that the Rh moments are weakly FM correlated with the nearest neighbor Fe moments. This is no doubt due to the explicit FM exchange between the Rh and Fe moments. In addition, Fig. 6(f) shows that for finite temperatures a weak FM correlation emerges between the nearest neighbor Rh moments in the AFMII phase. Thus within the AFMII phase there is a weak short-range signature of the magnetic structure of the FM phase. However, as expected, both $\left\langle\mathbf{M}_{\mathrm{Fe}}\right\rangle$ and $\left\langle\mathbf{M}_{\mathrm{Rh}}\right\rangle$ are zero for the AFMII phase.

The finite-temperature simulations involving 2000 moments for both the AFMII and FM phases did not undergo a phase change during the MC simulations. Although this result might be due to a simulation time-scale issue, it is compounded by the fact that for the present model only global volume-peratom fluctuations are admitted. For a volume-per-atom change that is of the magnitude necessary for the phase transition to occur, the associated energy change is extensive and therefore, from an energy perspective, inaccessible. While this result, the absence of a transition, is an artifact of the current model it does however demonstrate that volume plays a crucial role in the transition and infers local volume-per-atom fluctuations are required to obtain the transition. To investigate these aspects a much smaller sample is now considered of size $4 \times 4 \times 4$ containing only 128 moments. For this size of simulation cell, two values of Grueneisen parameter are used: (i) an average of the AFMII and FM values, $\gamma=(1.752+1.587) / 2$, and (ii) an increased value chosen to be $\gamma=(1.752+1.587) / 2+$ 1. The latter case will further enhance the global volume-peratom fluctuations. Thus, with these simulations volume-peratom fluctuations become larger at the expense of restricted moment fluctuations. In what follows, each simulation begins with the AFMII phase and for each temperature (50-600 K in steps of $50 \mathrm{~K}) 100 \times 10^{9} \mathrm{MC}$ steps are performed. All data shown and discussed (Figs. 7-9) will be for case (ii), the highest Gruneisen parameter. The results of case (i) will be discussed at the end of this section.

Figure 7(a) displays the Wigner-Seitz radii as a function of MC step for all temperatures considered. In this figure, the radii trajectory for each temperature is shifted upward to separate each curve for better inspection, where the lowest curve is at a temperature of $50 \mathrm{~K}$ and the uppermost curve is at a temperature of $600 \mathrm{~K}$. For the curves corresponding to temperatures less than $350 \mathrm{~K}$, no transition in volumeper-atom is evident. However at and above this temperature discrete changes in volume become increasingly frequent as the temperature rises. Inspection of the actual Wigner-Seitz radii regimes for each temperature reveals that the volumes accessible at each temperature are close to either that of the AFMII or FM structures seen in the larger cell-size simulations of Fig. 6. Indeed, at the lower temperatures, the system stays at a volume-per-atom similar to the expected volume-per-atom of the AFMII phase, and at the uppermost temperatures the system stays mainly in volume-per-atom corresponding to the FM phase. This may be seen in Fig. 7(b), which plots the average Wigner-Seitz radius of each temperature along with its standard deviation. In this figure the equilibrium data of Fig. 6(a) is also shown. This plot clearly shows a transition to volumes that may be associated with the FM magnetic structure, occurring at a temperature regime between 300 $400 \mathrm{~K}$. Away from this transition regime, the actual volumeper-atom is generally higher than that for the larger systems. These differences arise from the larger Gruneisen parameter used for case (ii). Despite this, the entire trend seen in Fig. 6(b) is qualitatively similar to that indicated by experimental thermal expansion measurements (compare with Fig. 1 in Ref. 32). 
To confirm that such volume fluctuations do in fact correspond to a change in magnetic structure, focus on the magnetic properties is now given to the circled region in Fig. 7(a) at 400 K. Figure 7(c) displays the corresponding volume-per-atom change of this region and, Fig. 7(d), the associated change in the $\mathrm{Fe}-\mathrm{Rh}, \mathrm{Fe}-\mathrm{Fe}$, and $\mathrm{Rh}-\mathrm{Rh}$ intersite moment correlation functions. The latter figure clearly indicates that at the volume change there is a change in magnetic structure from the AFMII to the FM phase, and that with respect to MC time step, the magnetic structure change and volume change occur in parallel. Further inspection of Figs. 7(c) and 7(d) also reveals an example of an unsuccessful excursion (occurring at MC step $22 \times 10^{9}$ ) from the AFMII phase in terms of magnetic structure and, to a lesser extent, volume.

Figure 8 now shows all these quantities for the temperatures of $350 \mathrm{~K}$ and $400 \mathrm{~K}$, for the entire MC simulation. Upon inspection of these figures, it becomes clear that whenever a volume change occurs there is indeed a corresponding change in magnetic structure. Moreover between these two temperatures a clear change in which phase is dominant is apparent. In Fig. 8(a), which displays the Wigner-Seitz radii
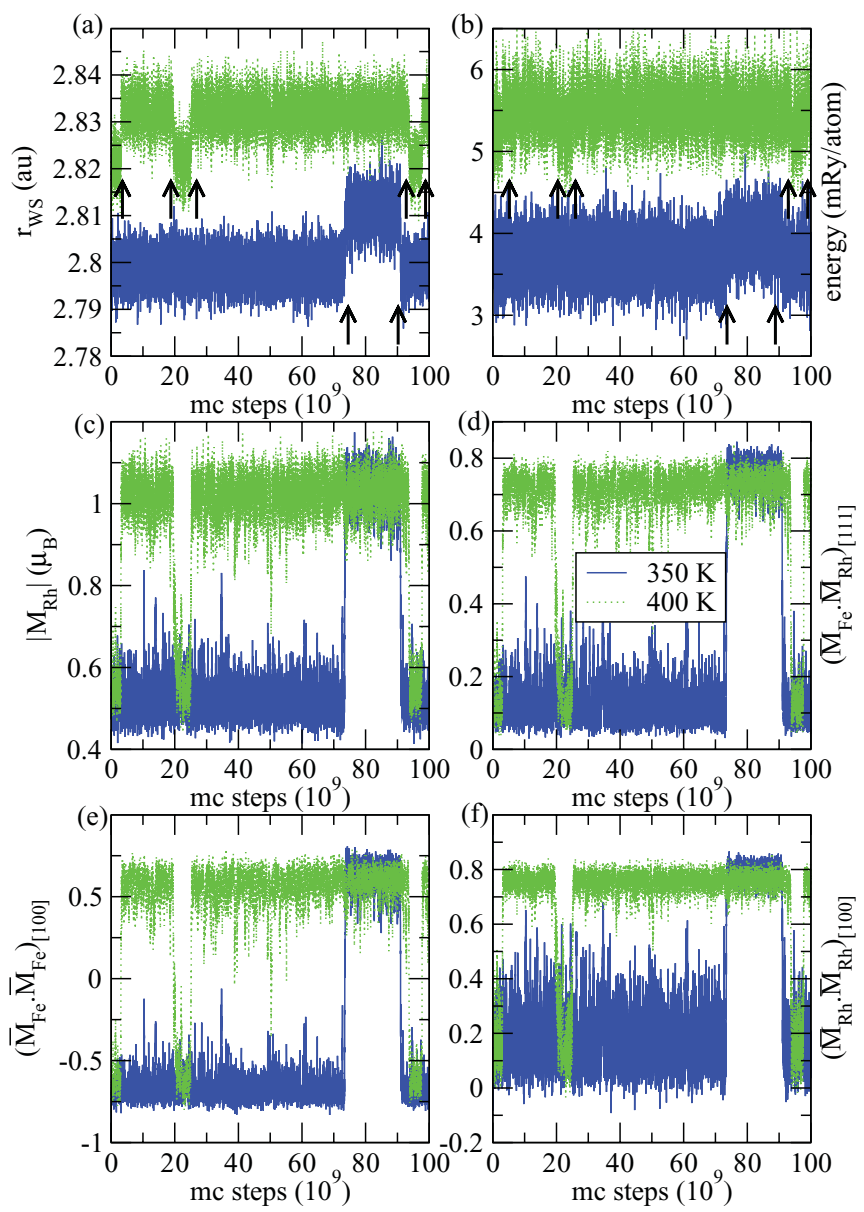

FIG. 8. (Color online) Plots of the $350 \mathrm{~K}$ and $400 \mathrm{~K}$ MC simulations undertaken in Fig. 7. Here (a) shows the Wigner-Seitz radii (where the $400 \mathrm{~K}$ value has been shifted up by $0.02 \mathrm{au}$ ), (b) the absolute energy (where the $400 \mathrm{~K}$ value has been shifted by $1 \mathrm{mRy}$ ), (c) the moment magnitude of the $\mathrm{Rh}$ atoms, and the normalized intersite correlation functions for the (d) FeRh, (e) FeFe, and (f) $\mathrm{RhRh}$ nearest neighbor moments. (with the $400 \mathrm{~K}$ radius shifted up by 0.02 atomic units), the regions undergoing a transition are indicated by arrows. Figure 8(b) now shows the absolute energy per atom (with the $400 \mathrm{~K}$ energy shifted up by $1 \mathrm{mRy}$ ) with the same transition regions again marked. What becomes clear is that the FM phase has a higher energy than the AFMII phase, even at $400 \mathrm{~K}$ where the system spends the majority of the simulation in the FM structure. At higher temperatures, changes in absolute energy cannot be detected within the fluctuations, nevertheless the system prefers to spend an increasing amount of the simulation in the FM phase.

The above results are a tentative signature of entropy stabilization of the FM phase due to fluctuations in both the moment and volume-per-atom degrees of freedom. In fact, inspection of Fig. 8(b) reveals an increase in internal energy of approximately $0.2 \mathrm{mRy} /$ atom when going from the AFMII to FM states, at the temperature range $350-400 \mathrm{~K}$. Within this range of temperatures there would exist a temperature, $T_{\mathrm{c}}$, at which the small system spends an equal amount of time in each magnetic phase-a situation in which the free-energy of both phases would be equal and $S_{\mathrm{FM}}\left(T_{\mathrm{c}}\right)-S_{\mathrm{AFMII}}\left(T_{\mathrm{c}}\right)=$ $\left(U_{\mathrm{FM}}\left(T_{\mathrm{c}}\right)-U_{\mathrm{AFMII}}\left(T_{\mathrm{c}}\right)\right) / T_{\mathrm{c}} \approx 0.2 \mathrm{mRy} / T_{\mathrm{c}}$. Allowing $T_{\mathrm{c}}$ to be in the range $350-400 \mathrm{~K}$ gives a corresponding specific entropy range of $8.2-9.4 \mathrm{JKg}^{-1} \mathrm{~K}^{-1}$ which compares reasonably with the measured change in entropy at the transition, ${ }^{10,14,33}$ $\Delta S^{\exp }=13-19.6 \mathrm{JKg}^{-1} \mathrm{~K}^{-1}$.

This somewhat crude approach can be given firmer grounding via determination of the specific heat. Figure 9(a) displays the corresponding specific heat as a function of temperature calculated via the fluctuations of the internal energy for both simulations cell sizes. In this figure, the Dulong-Petit limit value of the specific heat, $3 R / 2$ (where $R$ is the gas constant for the FeRh system in units of $\mathrm{JK}^{-1} \mathrm{Kg}^{-1}$ ) arising from the volume fluctuations is also shown. Relative to this value, the specific heat may be regarded as arising predominantly from the magnetic degrees of freedom. At the scale of the graph, the larger simulation cell shows that the AFMII and FM specific heats do not significantly differ (giving comparable linear specific heat coefficients in the range of $\sim 90$ $\mathrm{mJK}^{-2} \mathrm{Kg}^{-1}$, where experiment gives low-temperature values

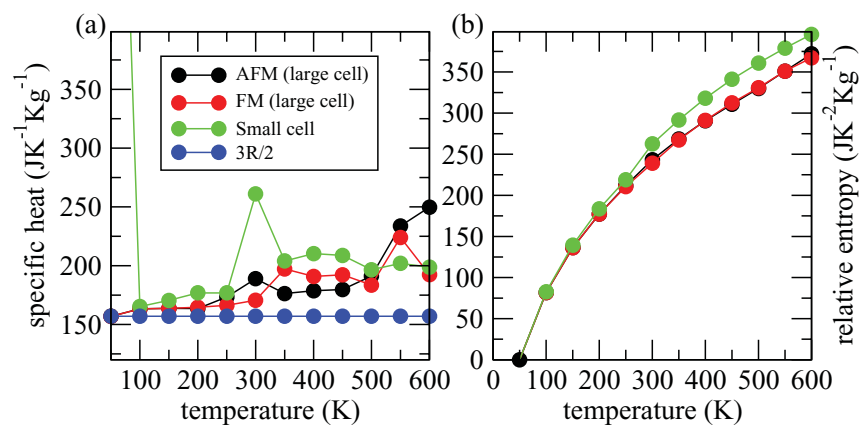

FIG. 9. (Color online) (a) specific heat as a function of temperature for the stable AFMII and FM phases using the $10 \times 10 \times 10$ simulation cell and for the smaller $4 \times 4 \times 4$ simulation cell in which volume fluctuations are enough to induce a magnetic transition. (b) Corresponding graphs of these three simulations for the entropy relative to their value at $50 \mathrm{~K}$ showing a jump in entropy as the smaller system transforms from the AFMII to the FM phase. 
of $10.5-16 \mathrm{mJK}^{-2} \mathrm{Kg}^{-1}$ for the AFM phase and 59-62.5 $\mathrm{mJK}^{-2} \mathrm{Kg}^{-1}$ for the FM phase), ${ }^{15,16}$ and due to the lack of a magnetic transition, no specific heat anomaly is evident in the temperature regime of $350-400 \mathrm{~K}$. On the other hand the specific heat derived from the smaller simulation cell, where local volume-per-atom fluctuations are now possible, does exhibit a clear peak at approximately $300 \mathrm{~K}$ and the specific heat of the low-temperature AFMII phase is lower by a factor of two than that of the high-temperature FM phase (relative to the Dulong-Petit limit). Figure 9(b) now displays the specific entropy change for each simulation with respect to the corresponding entropy at $50 \mathrm{~K}$. This is obtained from the specific heats of Fig. 9(a) via

$$
s(T)-s(50 \mathrm{~K})=\int_{50 \mathrm{~K}}^{T} d T \frac{c(T)}{T} .
$$

Inspection of this figure demonstrates that for the larger simulation cell, there is little difference between the stable AFMII and FM phases, however for the small simulation cell at approximately $300 \mathrm{~K}$, an anomalous increase in the entropy is evident corresponding to the AFMII/FM transition. This value is the order of $15 \mathrm{JKg}^{-1} \mathrm{~K}^{-1}$, which again compares well with experiment. That the transition is evident at the $300 \mathrm{~K}$ simulation rather than the $350 \mathrm{~K}$ simulation where the system clearly fluctuates between the two magnetic phases (Fig. 8) suggests, in part, precursor effects, but it might also be due to the relatively poor temperature resolution [and therefore integration variable in Eq. (29)] of the current simulations.

For case (i), in which the Gruneisen parameter is taken as the average of the AFMII and FM phases, transitions to the FM volume/magnetic structure also start to occur at $400 \mathrm{~K}$, however the system rapidly falls back to the AFMII structure. With increasing temperature, such events become more frequent, but with the system never spending a significant number of Monte Carlo steps in the FM structure, suggesting that for case (i), the volume fluctuations are not large enough to stabilize the higher-energy FM phase.

\section{DISCUSSION}

The central goal of the present work has been to develop an empirical model for the FeRh magnetic system that is able to describe both longitudinal and transverse moment fluctuations. This feature is necessary to reproduce the early colinear DFT work of Moruzzi and Marcus ${ }^{9}$ and the more contemporary noncolinear work of $\mathrm{Gu}$ and Antropov ${ }^{23}$ and Sandratskii and Mavropoulos. ${ }^{25}$ Using the Landau-Heisenberg model Hamiltonian this has been achieved using only nearest neighbor exchange parameters between the $\mathrm{Fe}$ and $\mathrm{Rh}$ atoms. In this sense the model is minimal. Indeed, it may be possible to better reproduce the DFT data via the addition of longer-range exchange interactions (the existence of which has been suggested by $\mathrm{DFT}^{23,25}$ ). That the current model is reasonably well able to reproduce the $a b$ initio derived behavior of the $0 \mathrm{~K}$ equilibrium magnetic phases supports such a minimal viewpoint, although inclusion of additional interactions will affect the dispersion properties of spin-wave excitations such as those considered by $\mathrm{Gu}$ and Antropov ${ }^{23}$ in the determination of their free energy contribution, and more recently discussed by Sandratskii and Buczek. ${ }^{34}$ Whether or not additional interactions are necessary to capture all that the first order transition entails, is a matter of further research.

In addition to the Landau-Heisenberg contribution, the final developed model includes a nearest neighbor quadratic exchange term, which stabilizes the FM phase with respect to transverse moment fluctuations. Historically, such an interaction has already emerged. In the exchange inversion model of Kittel, ${ }^{26}$ a similar term is derived when starting from a harmonic EOS plus a Heisenberg interaction between the magnetic sublattices (whose exchange constant depends linearly on the lattice constant). Here the equilibrium volume depends on the mean field exchange term, and when substituted back into the full energy expression results in the equation of state term being dependent on the square of the Heisenberg term. Even earlier, Lui et al. ${ }^{35}$ postulated the existence of such a term (via a $\cos 2 \theta$ angular dependence between the sublattice moments) as a phenomenological model for dysprosium, which also undergoes a AFM-FM phase transition at $85 \mathrm{~K}$. Such a model was found to better reproduce the experimental behavior than a model by Neel that relied on a complex sublattice-dependent crystalline anisotropy. ${ }^{36}$ As for a true microscopic understanding for $\mathrm{FeRh}$, it remains an open question as to whether this term may be justifiably associated with either the known quadratic exchange or Zener exchange mechanism, ${ }^{37-39}$ fundamental mechanisms that are normally associated with transition metal oxides.

An alternative mechanism to a quadratic exchange term could be to break the $O(3)$ symmetry of the Heisenberg Hamiltonian via the introduction of a cubic crystalline magneticanisotropy term for each $\mathrm{Fe}$ atom. A simple manifestation of this would be an energy term of the form

$$
E_{\text {Anisotropy }}=K\left(\alpha_{1}^{2} \alpha_{2}^{2}+\alpha_{2}^{2} \alpha_{3}^{2}+\alpha_{1}^{2} \alpha_{3}^{2}\right)
$$

where $\alpha_{1}=\cos \theta_{1}, \alpha_{2}=\cos \theta_{2}$, and $\alpha_{3}=\cos \theta_{3}$ constitute the direction cosines of a particular Fe moment with respect to the cartesian axes defined by the $\langle 100\rangle$ family of crystallographic directions. When the crystalline anisotropy constant, $K$, is positive, the $\langle 100\rangle$ are the easy axes and upon rotation of a moment from one axis to another, an energy barrier of $K / 4$ must be overcome. For BCC Fe, $K$ is typically $5 \times 10^{4}$ $\mathrm{J} / \mathrm{m}^{3}$ which corresponds to approximately $0.00026 \mathrm{mRy}$ per Fe atom. Transferal of this energy scale to the Fe moments in FeRh would therefore give an energy barrier of $2 \times K / 4 \simeq$ $0.00013 \mathrm{mRy}$ for the canting of the sublattice moments towards an orthogonal cartesian direction. This is significantly smaller than the energy barrier supplied by the quadratic Heisenberg term, $-J_{\mathrm{FeFe}}^{(2)}\left|\mathbf{M}_{\mathrm{Fe}}\right|^{4} \simeq 0.002 \times 3^{4}=0.162 \mathrm{mRy}$. Clearly, a considerably enlarged crystalline anisotropy would be needed to stabilize the the FM phase.

The simplicity of the current model affords an analytical investigation of the noncolinear magnetic moment variations performed in Sec. II B. In what follows, the quadratic exchange is not included. For the first noncolinear variation, the $\mathrm{Rh}$ moment of the FM configuration is rotated away from the $\mathrm{Fe}$ moment, where for each angle the stationary solution is 
obtained. Doing so, using Eq. (5), gives,

$$
E=-\frac{\left(B_{\mathrm{Fe}}+3 J_{\mathrm{FeFe}}-\frac{16 J_{\mathrm{FeRh}}^{2}}{B_{\mathrm{Rh}}} \cos ^{2} \theta\right)^{2}}{8 A_{\mathrm{Fe}}} .
$$

From this, the leading-order change in energy from the $\theta=0$ (FM) configuration is given by

$$
\begin{aligned}
\Delta E & \simeq-2 \frac{\left(B_{\mathrm{Fe}}+3 J_{\mathrm{FeFe}}-\frac{16 J_{\mathrm{FRh}}^{2}}{B_{\mathrm{Rh}}}\right)\left(\frac{16 J_{\mathrm{FeRh}}^{2}}{B_{\mathrm{Rh}}}\right)}{8 A_{\mathrm{Fe}}} \sin ^{2} \theta \\
& \simeq-4 \frac{B_{\mathrm{Fe}}}{A_{\mathrm{Fe}}} \frac{J_{\mathrm{FeRh}}^{2}}{B_{\mathrm{Rh}}} \sin ^{2} \theta .
\end{aligned}
$$

For the parameters of the model, this turns out to be a good approximation to the energy variation shown in Fig. 4(b) over the entire considered volume range because $3 J_{\mathrm{FeFe}}-16 J_{\mathrm{FeRh}}^{2} / B_{\mathrm{Rh}}$ is quite small compared to $B_{\mathrm{Fe}}$. The corresponding $\mathrm{Rh}$ moment magnitude is given by

$$
\begin{aligned}
M_{\mathrm{Rh}} & =-\frac{8 J_{\mathrm{FeRh}} \cos \theta}{B_{\mathrm{Rh}}} \sqrt{\frac{B_{\mathrm{Fe}}+3 J_{\mathrm{FeFe}}-\frac{16 J_{\mathrm{FeRh}}^{2}}{B_{\mathrm{Rh}}} \cos ^{2} \theta}{8 A_{\mathrm{Fe}}}} \\
& \simeq-\frac{\sqrt{8} J_{\mathrm{FeRh}} \cos \theta}{B_{\mathrm{Rh}}} \sqrt{\frac{B_{\mathrm{Fe}}}{A_{\mathrm{Fe}}}}
\end{aligned}
$$

Substitution of Eq. (35) into Eq. (33) gives the result

$$
\Delta E \simeq-\frac{1}{2} B_{\mathrm{Rh}}\left\{\left[M_{\mathrm{Rh}}(\theta=0)\right]^{2}-\left[M_{\mathrm{Rh}}(\theta)\right]^{2}\right\} .
$$

In choosing a positive value for $B_{\mathrm{Rh}}$, it is from this perspective that Ju et al. ${ }^{17}$ have argued a Stoner-like form to describe the energy contribution of a finite $\mathrm{Rh}$ moment.

The second and third colinear variations performed in Sec. II B are also easily obtainable. Specifically, for the Fe canting variation in which the $\mathrm{Rh}$ moment is fixed to be parallel to the total Fe moment [Figs. 4(c) and 4(d)] the corresponding energy as a function of canting angle is given by

$$
E=-\frac{\left(B_{\mathrm{Fe}}-3 J_{\mathrm{FeFe}} \cos 2 \theta-\frac{J_{\mathrm{FeRh}}^{2}}{B_{\mathrm{Rh}}} \sin ^{2} \theta\right)^{2}}{8 A_{\mathrm{Fe}}},
$$

whereas the energy associated with an Fe canting, in which the $\mathrm{Rh}$ moment magnitude is constrained to be zero [Fig. 4(e)] is given by

$$
E=-\frac{\left(B_{\mathrm{Fe}}-3 J_{\mathrm{FeFe}} \cos 2 \theta\right)^{2}}{8 A_{\mathrm{Fe}}}
$$

Obtaining similar leading order forms of Eqs. (37) and (38) results in their difference equaling the negative of Eq. (33). Thus the assertion of Ref. 25 that the second colinear variation probes a mixture of the the FeFe and FeRh exchange interactions, and the third colinear variation probes only the FeFe exchange interaction, is supported by the present model where their difference yields directly an effective FeRh interaction of the form given by Eq. (33).

Despite the coupled Landau and Heisenberg contributions to the energy, it is possible to perform a direct comparison of the fitted parameters with other work since factors involving ratios of the Fe Landau parameters can be rewritten as Fe moment magnitudes, which when multiplied by the appropriate exchange parameters will yield an interaction energy. Inspection of Eq. (33) gives a prefactor that varies between 7.9 and $9.7 \mathrm{mRy}$ (over the volumes considered) or an effective exchange interaction between the $\mathrm{Fe}$ and $\mathrm{Rh}$ moments of 1-1.2 mRy per bond. This compares quite well to the exchange parameter, $J_{\mathrm{FeRh}}=1.2 \mathrm{mRy}$ of Sandratskii and Mavropoulos. ${ }^{25}$ Via Eq. (36), Eq. (33) may also be viewed entirely from the perspective of the Landau picture, giving an onsite energy for the $\mathrm{Rh}$ atom of 8.1-9.1 (evaluated at a $\mathrm{Rh}$ moment magnitude of one Bohr magneton), which is in reasonable agreement with the value of $\approx 6 \mathrm{mRy}$ found by Sandratskii and Mavropoulos. ${ }^{25}$ Insight into the effective exchange between the nearest neighbor FeFe moments is found by considering the leading-order variation of Eq. (38)

$$
\Delta E \simeq-\frac{3}{2} \frac{B_{\mathrm{Fe}}}{A_{\mathrm{Fe}}} J_{\mathrm{FeFe}} \sin ^{2} \theta .
$$

Here the prefactor varies between 9.4 and $9.9 \mathrm{mRy}$ corresponding to approximately $1.6 \mathrm{mRy}$ per bond, which is somewhat higher than the shell-averaged exchange interaction of Ref. 25 derived from their Fig. 9.

The current model only admits global volume-per-atom fluctuations, and the present work appears to indicate that because of this, the bulk magnetic phase transition cannot directly be studied. It may be thus inferred that local volumeper-atom fluctuations play a crucial role in the transition from one magnetic structure to the other. This assertion is supported by the results of the $4 \times 4 \times 4$ cell simulations, where due to the small global volume-per-atom and larger Grüneisen parameter, global volume-per-atom fluctuations are enhanced sufficiently enough to allow the system to fluctuate between the AFMII and FM structures in the temperature regime of approximately 300-400 K, and stay predominantly in the FM structure at higher temperatures. This occurs despite the increase in internal energy associated with transforming to the FM phase, suggesting the important role of entropy arising from the volume and/or moment degrees of freedom. Although the simulation is in some sense artificial, this is nevertheless a revealing result and quantitatively consistent with the large entropy measured at the transition. ${ }^{10,14,33}$

The current work therefore hints at the comparable role both the magnetic and volume-per-atom degrees of freedom play in the transformation of FeRh from the AFM to FM phase. To investigate these issues experimentally, a new generation of so-called pump-and-probe experiments have been able to resolve the temporal properties of the first-order transition to near picosecond resolution. In such experiments, the pump illuminates the FeRh thin film with a fluence sufficient to raise the temperature above the transition temperature, and the probe measures the magnetization dynamics, usually, via the optical Kerr effect. In the work of Ju et al., ${ }^{17}$ both the change in polarization and the reflectivity were measured. Here the polarization gave information about the total magnetization and was found to increase rapidly from zero (at the time of the pump) to a saturation value in a timescale of approximately 50 picoseconds, after which it decreased at a much longer timescale. This could be directly interpreted as the transformation from the AFM phase to the FM phase, and the corresponding emergence of a total magnetization, which eventually reduces due to subsequent cooling of the FeRh system. Later work by Bergman et al. ${ }^{18}$ demonstrated that 
the magnetization dynamics could be understood by reorientation dynamics due to the initially nonaligned FM domains aligning to a single homogeneous domain state. In Ref. 17, by subtracting from the reflectivity a transient electronic component, the authors could also obtain information on lattice expansion. This indirect measure suggested a volume increase that occurred somewhat faster than the overall magnetization response. Similar experiments and conclusions were obtained by Theile et al. ${ }^{19}$

More recent experiments tend to confirm the sub-100 picosecond timescale of the AFM-FM transformation. Radu et $a l .^{21}$ used time-resolved $\mathrm{x}$-ray magnetic circular dichroism measurements to probe the magnetization dynamics and confirmed that the timescale for the creation of the FM magnetic structure was indeed in the sub-100 picosecond timescale. Quirin et al., ${ }^{20}$ performed pump-and-probe experiments using time-resolved XRD to directly measure structure via the time evolution of a Bragg peak. They found that lattice expansion occurred at a timescale of 100 picoseconds, however their experiments did not measure the corresponding magnetic evolution.

Mariager et al., ${ }^{22}$ performed similar experiments that now directly probed the lattice constant of the FeRh thin film via XRD and the time evolution of the magnetization via the optical Kerr effect. They found that after the pump, a new Bragg peak emerges at the expense of the original Bragg peak, giving a clear indication of the coexistence of two lattice constants within the thin film, and tentative evidence for the coexistence of both the AFM and FM phases, a central hallmark of a first-order phase transition. Thus, from the Bragg peak measurements quantitative temporal information on the FM volume fraction was obtained. The data indicated a rapid rise and saturation of the FM volume fraction with a characteristic timescale of $\simeq 14$ picoseconds. However, the optical Kerr signal that measured total magnetization exhibited a distinctly different response in both form and (longer) timescale. By asserting that FM regions nucleated locally with a total magnetic moment along either of four directions (orthogonal to the thin film normal), resulting in an early zero net magnetization, the slower magnetization increase could be understood from the perspective of magnetic domain alignment dynamics. Doing so resulted in a magnetic domain nucleation time scale of $\simeq 18$ picoseconds, which is comparable to their XRD derived structural nucleation time scale. From this the authors concluded that within their error bars, the magnetic and volume nucleation timescales associated with the first-order AFM to FM phase transition are the same.

The proceeding discussion motivates modifications to the present model to explicitly include local volume fluctuations. How may this be achieved? In terms of the magnetic energy contribution to the total cohesive energy, the global WignerSeitz parameter can be straightforwardly taken as a local parameter, entailing that the exchange parameters depend on the appropriate interatomic distance and that the on-site Landau terms become some function of local volume, again via the nearest neighbor environment defined by the appropriate interatomic distances. In the same spirit, the nonmagnetic contribution to the cohesive energy can be described by an empirical interatomic force model that correctly describes the nonmagnetic contribution to elasticity, thermal expansion, and equilibrium volume. Indeed, for pure FM BCC Fe, the nonmagnetic and Landau contributions to the equilibrium cohesive energy have been modeled in unified way via the so-called magnetic potential formalism, ${ }^{40,41}$ in which the local volume needed by the Landau term is effectively obtained via the environmental dependence of the second moment of the local electronic DOS. Inclusion of transverse moment fluctuations into the $\mathrm{BCC} F$ system is then done via the addition of a Heisenberg term, constituting the so-called spin lattice dynamics technique, which is able to model both lattice and moment degrees of freedom in BCC Fe. ${ }^{42,43}$ In general terms such an atomistic approach has also been partially followed in the work of Gruner et al., ${ }^{27}$ enabling them to model local volume fluctuations and therefore the actual AFM-FM phase transition. An alternative, somewhat simpler, approach is to couple the magnetic degrees of freedom to a continuum description of an appropriate elastic medium in which local (to chosen length scale) variations in volume are admitted. For the present Landau-Heisenberg model, these considerations will be actively pursued in future work.

\section{CONCLUSION}

In conclusion, an empirical model for the FeRh system has been developed within the framework of a volume-per-atom dependent Landau-Heisenberg Hamiltonian, allowing for both longitudinal and transverse fluctuations in the magnetic moments of both the $\mathrm{Fe}$ and $\mathrm{Rh}$ atoms. Upon fitting to past colinear and noncolinear DFT calculations of FeRh, the model is able to reasonably well reproduce a wide range of $a b$ initio derived trends and quantities. It is however found that in order to stabilize the ferromagnetic state with respect to transverse moment fluctuations, a quadratic exchange term must be added to the Hamiltonian, which is similar in form to the well-known double-exchange mechanism. A nonmagnetic contribution to energy is also added to the Hamiltonian via a nonmagnetic equation of states curve, thus allowing the model to admit global volume-per-atom fluctuations. Via Monte Carlo simulation, the thermal properties of both phases were determined giving thermal expansion coefficients and Curie/Neel temperatures that agree reasonably well with experiment. For large system sizes, no antiferromagnetic to ferromagnetic transition was observed inferring the important role of local volume fluctuations, a feature not included in the current model. This aspect was further investigated by considering smaller simulation cells at finite temperature, a regime where the energy of global volume-per-atom fluctuations is comparable to that of the magnetic moment fluctuations. In this case, the antiferromagnetic to ferromagnetic transition was observed in the temperature range of 300-400 K and at higher temperatures the ferromagnetic phase was stabilized. The corresponding entropy change due to the transition compared well to experiment. The present model therefore suggests the comparable importance of both volume-per-atom and magnetic moment degrees of freedom as driving forces for the antiferromagnetic to ferromagnetic transition occurring in the FeRh system. 


\section{ACKNOWLEDGMENTS}

The author thanks G. Ingold, S. O. Mariager, and C. Quitmann for introduction to the FeRh system and for many useful discussions, and also S. L. Dudarev for extensive discussions on the Landau-Heisenberg modeling framework. *peter.derlet@psi.ch

${ }^{1}$ M. Fallot, Ann. Phys. (Paris) 10, 291 (1938).

${ }^{2}$ M. Fallot and R. Hocart, Rev. Scient. 77, 498 (1939).

${ }^{3}$ J. S. Kouvel and C. C. Hartelius, J. Appl. Phys. Suppl. 33, 1343 (1962).

${ }^{4}$ F. de Bergevin and L. Muldawer, Compt. Rend. 252, 1347 (1961).

${ }^{5}$ J.-U. Thiele, S. Maat, J. L. Robertson, and E. E. Fullerton, IEEE Trans. Magn. 40, 2537 (2004).

${ }^{6}$ G. Shirane, R. Nathans, and C. W. Chen, Phys. Rev. 134, A1547 (1964).

${ }^{7}$ C. Koenig, J. Phys. F 19, 1123 (1982).

${ }^{8}$ J. Hasegawa, J. Magn. Magn. Mater. 66, 175 (1987).

${ }^{9}$ V. L. Moruzzi and P. M. Marcus, Phys. Rev. B 46, 2864 (1992).

${ }^{10}$ J. S. Kouvel, J. Appl. Phys. 37, 1257 (1966).

${ }^{11}$ A. I. Zakharov, A. M. Kadomtseva, R. S. Levitin, and E. G. Ponyatovskii, Zh. Eksp. Teor. Fiz. 46, 2003 (1964) [Sov. Phys. JETP 19, 1348 (1964)].

${ }^{12}$ J. M. Lommel, J. Appl. Phys. 40, 3880 (1969).

${ }^{13}$ J. B. McKinnon, D. Melville, and E. W. Lee, J. Phys. C 3, S46 (1970).

${ }^{14}$ M. P. Annaorazov, S. A. Nikitin, A. L. Tyurin, K. A. Asatryan, and A. K. Dovletov, J. Appl. Phys. 79, 1689 (1996).

${ }^{15}$ P. Tu, A. J. Heeger, J. S. Kouvel, and J. B. Comly, J. Appl. Phys. 40, 1368 (1969).

${ }^{16}$ J. Ivarsson, G. R. Pickett, and J. Toth, Phys. Lett. A 35, 167 (1971).

${ }^{17}$ G. Ju, J. Hohlfeld, B. Bergman, R. J. M. van deVeerdonk, O. N. Mryasov, J.-Y. Kim, X. Wu, D. Weller, and B. Koopmans, Phys. Rev. Lett. 93, 197403 (2004).

${ }^{18}$ B. Bergman, G. Ju, J. Hohlfeld, R. J. M. van de Veerdonk, J.-Y. Kim, X. Wu, D. Weller, and B. Koopmans, Phys. Rev. B 73, 060407(R) (2006).

${ }^{19}$ J.-U. Thiele, M. Buess, and C. H. Back, Appl. Phys. Lett. 85, 2857 (2004).

${ }^{20}$ F. Quirin, M. Vattilana, U. Shymanovich, A.-E. El-Kamhawy, A. Tarasevitch, J. Hohlfeld, D. von der Linde, and K. SokolowskiTinten, Phys. Rev. B 85, 020103(R) (2012).

${ }^{21}$ I. Radu, C. Stamm, N. Pontius, T. Kachel, P. Ramm, J.-U. Thiele, H. A. Dürr, and C. H. Back, Phys. Rev. B 81, 104415 (2010).
${ }^{22}$ S. O. Mariager, F. Pressacco, G. Ingold, A. Caviezel, E. MöohrVorobeva, P. Beaud, S. L. Johnson, C. J. Milne, E. Mancini, S. Moyerman, E. E. Fullerton, R. Feidenhans'l, C. H. Back, and C. Quitmann, Phys. Rev. Lett. 108, 087201 (2012).

${ }^{23}$ R. Y. Gu and V. P. Antropov, Phys. Rev. B 72, 012403 (2005).

${ }^{24}$ B. K. Ponomarev, Sov. Phys. JETP 26, 105 (1973).

${ }^{25}$ L. M. Sandratskii and P. Mavropoulos, Phys. Rev. B 83, 174408 (2011).

${ }^{26}$ C. Kittel, Phys. Rev. 120, 335 (1960).

${ }^{27}$ M. E. Gruner, E. Hoffmann, and P. Entel, Phys. Rev. B 67, 064415 (2003).

${ }^{28}$ L. D. Landau, Phys. Z. Sowjetunion 11, 26 (1937) [Collected Papers of L. D. Landau, edited by D. ter Haar (Pergamon Press, Oxford, 1965), pp. 193-215].

${ }^{29}$ E. C. Stoner, Proc. R. Soc. A 169, 339 (1939).

${ }^{30}$ J. Friedel, Adv. Phys. 3, 446 (1954).

${ }^{31}$ A. Corana, M. Marchesi, C. Martini, and S. Ridella, ACM Trans. Math. Softw. 13, 262 (1987).

${ }^{32}$ M. R. Ibarra and P. A. Algarabel, Phys. Rev. B 50, 4196 (1994).

${ }^{33}$ M. J. Richardson, S. A. Nikitin, A. L. Tyurin, K. A. Asatryan, and A. Kh. Dovletov, Phys. Lett. 46, 153 (1973).

${ }^{34}$ L. M. Sandratskii and P. Buczek, Phys. Rev. B 85, 020406(R) (2012).

${ }^{35}$ S. H. Liu, D. R. Behrendt, S. Legvold, and R. H. Good Jr., Phys. Rev. 116, 1464 (1959).

${ }^{36}$ L. Neel, Compt. rend. 242, 1549 (1956); 242, 1824 (1956).

${ }^{37}$ C. Zener, Phys. Rev. 82, 403 (1951).

${ }^{38}$ P. W. Anderson and H. Hasegawa, Phys. Rev. 100, 675 (1955).

${ }^{39}$ P. G. De Gennes, Phys. Rev. 118, 141 (1960).

${ }^{40}$ S. L. Dudarev and P. M. Derlet, J. Phys.: Condens. Matter 17, 7097 (2005).

${ }^{41}$ P. M. Derlet and S. L. Dudarev, Prog. Mater. Sci. 52, 299 (2007).

${ }^{42}$ S. L. Dudarev and P. M. Derlet, J. Comput,-Aided Mater. Des. 14, 129 (2007).

${ }^{43}$ P.-W. Ma, C. H. Woo, and S. L. Dudarev, Phys. Rev. B 78, 024434 (2008). 